\title{
Polarimetric Response of Rice Fields at C-Band: Analysis and Phenology Retrieval
}

\author{
Juan M. Lopez-Sanchez, Senior Member, IEEE, Fernando Vicente-Guijalba, \\ J. David Ballester-Berman, and Shane R. Cloude, Fellow, IEEE
}

\begin{abstract}
A set of ten Radarsat-2 images acquired in fully polarimetric mode over a test site with rice fields in Seville, Spain, has been analysed to extract the main features of the $\mathrm{C}$-band radar backscatter as a function of rice phenology. After observing the evolutions vs phenology of different polarimetric observables and explaining their behaviour in terms of scattering mechanisms present in the scene, a simple retrieval approach has been proposed. This algorithm is based on three polarimetric observables and provides estimates from a set of four relevant intervals of phenological stages. The validation against ground data, carried out at parcel level for a set of 6 stands and up to 9 dates per stand, provides a $96 \%$ rate of coincidence. Moreover, an equivalent compact-pol retrieval algorithm has been also proposed and validated, providing the same performance at parcel level. In all cases, the inversion is carried out by exploiting a single satellite acquisition, without any other auxiliary information.
\end{abstract}

Keywords—Synthetic aperture radar, agriculture, polarimetry, rice, phenology

\section{INTRODUCTION}

Rice is surely the most important crop in the world because it constitutes the staple food for more than half of the total population. Attending to the increasing food demand and other economical, social, and also environmental issues related with its cultivation, there is a significant interest in the information provided by remote sensing about rice fields. Among other sensors, radar instruments are especially well suited for such purposes thanks to their all-weather operation and day/night independence.

In the past, successful results were obtained with radar satellites operating at C-band (ERS, Radarsat) and L-band (JERS), demonstrating that mapping rice-planted areas and monitoring its evolution with backscatter coefficients at $\mathrm{HH}$ or VV channels was feasible [1], [2], [3], [4]. The C-band HH/VV ratio, provided by Envisat-ASAR in AP mode, was also used to discriminate cultivated fields from non-rice fields, showing a variation up to $4-7 \mathrm{~dB}$ from the beginning of the season to the plant maturity phase [5]. Experiments with X-band satellite data, provided by TerraSAR-X, for studying the evolution of rice fields were also conducted more recently [6]. There exist also some examples of studies over rice fields carried out with airborne experimental sensors [7] and ground-based scatterometers [8].

Besides the interest in applications such as rice mapping and rice production estimation, which have concentrated most studies to date, precision farming can be also tackled for rice with the help of radar satellites. This is possible especially

J. M. Lopez-Sanchez, J. D. Ballester-Berman and F. Vicente-Guijalba are with the Institute for Computing Research (IUII), University of Alicante, P.O.Box 99, E-03080 Alicante, Spain (e-mail: juanma-lopez@ieee.org, fernando.vicente@ua.es, davidb@ua.es). Shane R. Cloude is with AEL Consultants, 26 Westfield Avenue, Cupar, Fife, KY15 5AA, Scotland, UK (e-mail: aelc@mac.com). This work was supported by the Spanish Ministry of Economy and Competitiveness (MINECO) and EU FEDER, under Project TEC201128201-C02-02. since the launch of satellites with short revisit times (11 days for TerraSAR-X) and/or with enhanced observation capabilities provided by their polarimetric modes (ALOS-PALSAR, Radarsat-2, TerraSAR-X). The primary objective of remote sensing applied to precision farming consists in providing timely and accurate information about the crop condition, at reduced local scale, in order to plan or trigger cultivation practices (irrigation, fertilisation, etc.) accordingly. To this end, the final users (farmers or farm managers) are interested in knowing the current situation of the crop along its cultivation cycle, i.e. the current phenological stage at the time of observation.

In the case of rice fields, there are several examples of key moments that should be identified. For instance, at the beginning of the tillering stage, once all plants have emerged (if plantation is carried out by sowing, not by transplanting) and are starting to develop, the number or density of plants effectively emerged is measured and compared with the number of seeds. This measurement is called effective germination. If it is too low, a second round of sowing is practised. Another important moment is the beginning of the heading stage, since from that moment it is recommended stopping fertilisation. Otherwise, pests could appear more easily. In addition to specific stages, farmers are very interested in detecting phenological delays, which may appear as a consequence of other factors, such as plagues, water salinity, etc.

The estimation of phenology of rice fields by exploiting satellite SAR data was firstly addressed in [9], using coherent dualpol HHVV images from TerraSAR-X. A simple algorithm based on polarimetric observables provided by a single acquisition was designed to retrieve the phenological stage from a set of five relevant intervals. Results showed the sensitivity of polarimetry to the phenology of rice, obtaining successful estimates in most cases. Nevertheless, some ambiguities were also identified, i.e. two different stages might produce the same radar response in the available observation space.

In this work we have applied a similar approach but to a set of fully polarimetric images acquired by Radarsat- 2 over the same test site. A comparison between the observations provided by both datasets, gathered at different frequencies, will be commented along the text. However, the first goal of this work is checking whether the additional information provided by full polarimetry can complement the previous dual-pol observations or not, especially for solving the mentioned ambiguities. To this end, an analysis of the Radarsat-2 data as a function of phenology has been carried out, employing a large number of observables (backscattering coefficients, decompositions, etc.).

A second objective of this study, aside from the complementarity with the previous work, consists in exploring the potential 
of C-band sensors for rice phenology retrieval. In particular, due to the long revisit time of Radarsat-2 (24 days), we have studied the application of the phenology retrieval algorithm to single acquisitions, i.e. ignoring any other temporal or auxiliary information.

Finally, we have tested the performance in this particular application of other polarimetric modes of reduced dimensionality (compact and dual polarimetry) with the aim of anticipating results from the future Canadian Radarsat Constellation Mission (RMC) and the European Sentinel-1 system.

There exist in the literature other two examples of Radarsat2 data applied to rice observation. In the first case, Yang et al. [10] analysed a set of only two images acquired at different phenological stages, and their analysis was restricted only to the backscattering coefficients. In a second work, Li et al. [11] disposed of four images starting at the reproductive phase. They employed a more elaborate analysis, including decompositions, but their main goal was rice mapping, not phenology retrieving. In our case, the dataset comprises 10 images and covers a wider phenological range, so it can be considered as the most complete to date.

The text is organised as follows. The test site and the data from the ground campaign and the radar acquisitions are presented in Section II. Then, the polarimetric observations are analysed as a function of phenology in Section III. Section IV describes a phenology retrieval approach and its validation against ground reference data. The main conclusions are summarised in Section V.

\section{TEST SITE, GROUND MEASUREMENT CAMPAIGN AND SAR DATA}

The test site consists of an area of $30 \mathrm{~km} \times 30 \mathrm{~km}$ in the mouth of the Guadalquivir river, Seville, SW of Spain, where rice is cultivated annually from May to October, approximately. An RGB composite of this area, obtained from a Radarsat- 2 image acquired on 6-August-2010, is shown in Figure 1 for reference. General rice species in this area is Oryza sativa L.. The specific variety cultivated in the monitored fields corresponds to a long grain type named puntal, quite common in Spain and other similar temperate regions.

Cultivation practises are very diverse all over the world. For instance, cultivation may be initiated by transplanting, wet sowing or dry sowing, and then fields can be drained during the cultivation cycle or not. Consequently, growth cycle can be very short (e.g. 80 days) or much longer (up to 150 days) depending on the practises and the region. In this specific location, sowing is carried out by spreading seeds randomly from an airplane over the fields, which are already flooded at that time. Then, farming practises in this area ensure the presence of a water layer on the ground during the whole cultivation period, hence the ground is always flooded and will be considered as a water surface from the radar point of view. Finally, the cultivation campaign lasts about 135-150 days.

Since 2008, the local association of rice farmers (Federacion de Arroceros de Sevilla) has collected detailed ground measurements on a weekly basis. For this research project, centred in the 2009 and 2010 campaigns, five specific parcels, spread over the whole site, were selected for intensive sampling during 2009,

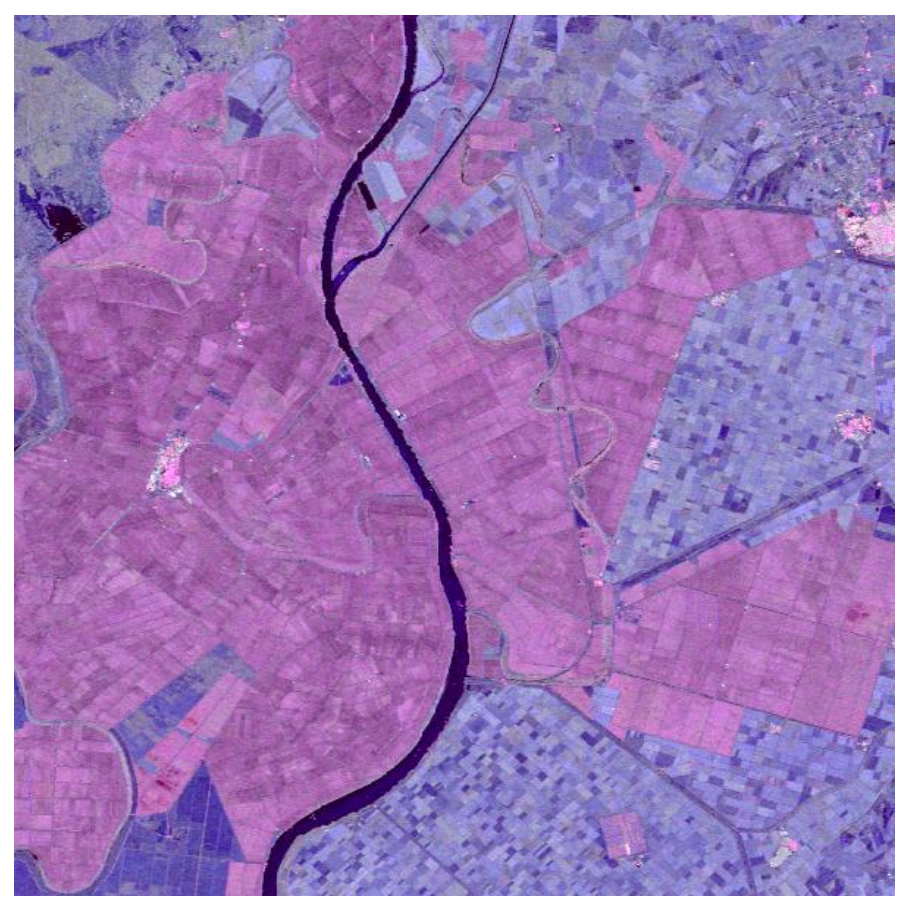

Fig. 1. RGB composite of the test site obtained in the Pauli basis from a Radarsat-2 image acquired on 6-August-2010. Colour coding: $\mathrm{Red}=\mathrm{HH}$ $\mathrm{VV}$, Green $=2 \mathrm{HV}$, and Blue $=\mathrm{HH}+\mathrm{VV}$. All channels are obtained as calibrated backscattering coefficients and mapped in the same range: Minimum $=-30 \mathrm{~dB}$, and Maximum $=-5 \mathrm{~dB}$. Values exceeding these limits are saturated to the extreme values. Rice fields are clearly identified in pink colour.

adding a new one in 2010. The weekly measurements include phenological stage and total vegetation height.

A description of the phenological evolution of rice can be found in other references [12] and was already summarised in our previous work [9], so only a few important remarks will be repeated here for clarity purposes. Three main stages are usually defined for the rice growth cycle: vegetative, reproductive and maturation or ripening, each of them with particular features and aspects of the plant development as a function of time. This temporal evolution is described numerically by identifying 10 main stages (assigned to tens) which are subsequently subdivided into 10 secondary stages (assigned to units), hence resulting in a continuous numerical scale of the plant phenology from 0 to 100. A widely employed scale of such type is the general BBCH scale (from Biologische Bundesanstalt, Bundessortenamt und CHemische Industrie) for cereals [13], [12], which provides also a particular representation for rice. The first half of the cycle, from stage 0 to 49 , corresponds to the mentioned vegetative phase, whereas stages 50 to 69 and 70 to 99 constitute the reproductive and maturation phases, respectively.

The description in terms of the $\mathrm{BBCH}$ scale is based on the actual characteristics of an individual plant. When the scale is used for the definition of the development stage of a plant stand or a parcel or a field, the description should apply to at least $50 \%$ of the plants inside the parcel. Therefore, a specific field or parcel will be considered to reach a particular phenological stage when more than half of its plants reach that stage. This criterion was employed in the ground campaign and will be taken into account when analysing the relationship between ground 

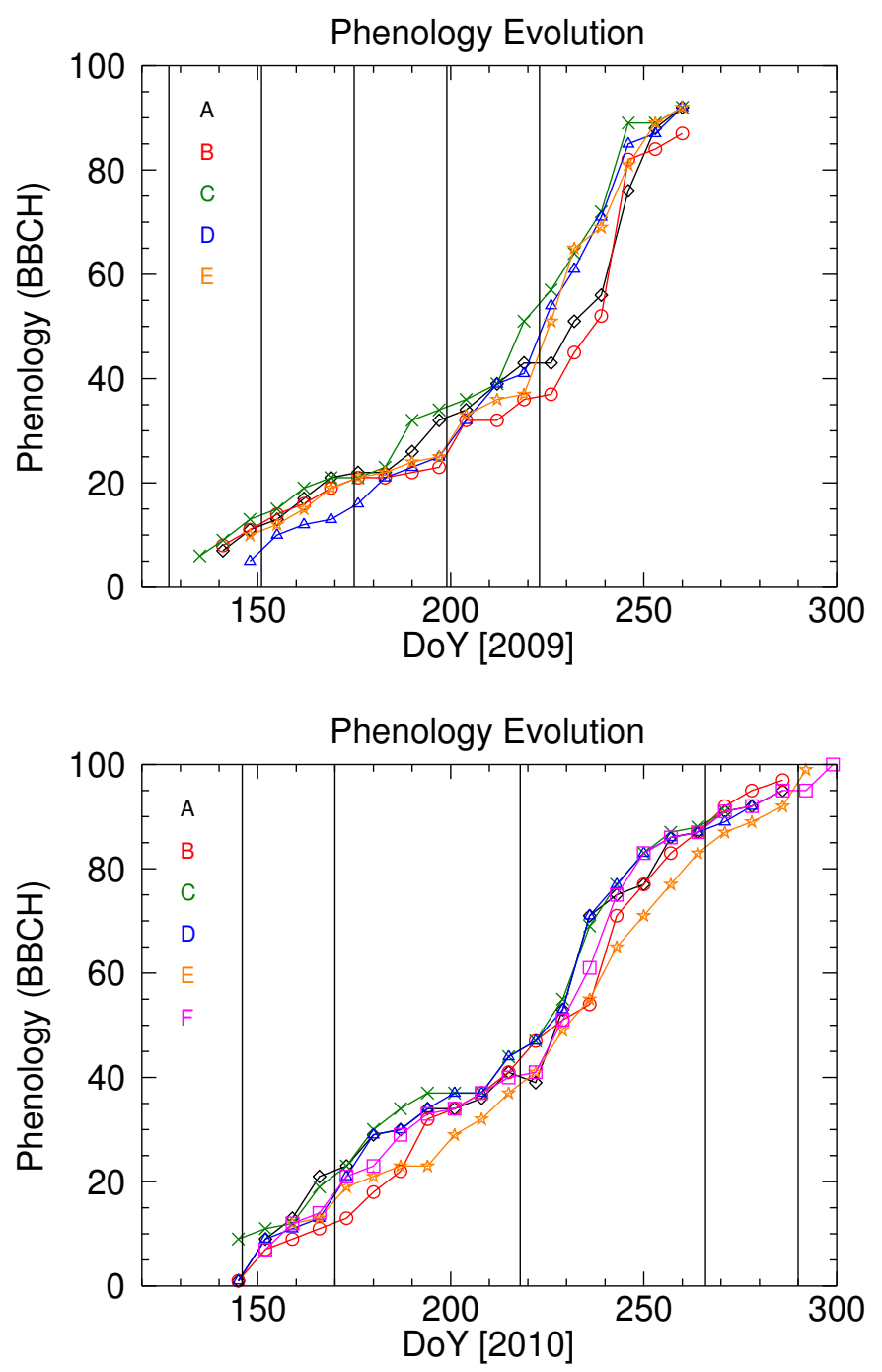

Fig. 2. Evolution of the phenology at the monitored parcels as acquired during the ground campaign, plotted as a function of day of year (DoY). Top: 2009, Bottom: 2010. Vertical black lines denote the dates of acquisition of the Radarsat-2 images.

measurements and radar observables. A clear consequence is that plant development may be quite heterogeneous inside some parcels at some stages of the campaign.

In addition to phenology, the following information is known for each parcel (see Table I): total area (ha), sowing date, surface density of plants (plants $/ \mathrm{m}^{2}$ ) and panicles (panicles $/ \mathrm{m}^{2}$ ), harvest date, and final yield ( $\mathrm{kg} / \mathrm{ha}$ ). Particular aspects for some of them have been registered also, such as irrigation conditions, water salinity and presence of plagues. Note that neither sowing nor harvest are simultaneous in all parcels of the site, being around 3 to 4 weeks time span between the first and the last of the monitored parcels in both activities.

Finally, there is also climate information provided by the Spanish Government under the Sistema de Informacion Agroclimatica para el Regadio (SIAR), including daily files of temperature, precipitation, humidity and wind. In this region, a rainy season is common at the beginning of autumn every year and did not affect the radar acquisitions available in this study.
TABLE II

LIST OF AVAILABLE RADARSAT-2 IMAGES

\begin{tabular}{cccc}
\hline Acquistion date & DoY (2009) & Acquistion date & DoY (2010) \\
\hline 07-May-2009 & 127 & 26-May-2010 & 146 \\
31-May-2009 & 151 & 19-Jun-2010 & 170 \\
24-Jun-2009 & 175 & 06-Aug-2010 & 218 \\
18-Jul-2009 & 199 & 23-Sep-2010 & 266 \\
11-Aug-2009 & 223 & 17-Oct-2010 & 290 \\
\hline
\end{tabular}

TABLE III

INFORMATION ABOUT THE RADARSAT-2 SLC IMAGES

\begin{tabular}{lc}
\hline Mode & Fine Quad-Polarisation \\
Beam & FQ13 \\
Avg. Incidence angle & 33 degrees \\
Orbits & Descending \\
Time of acquisition & $6: 30 \mathrm{am}$ \\
Centre frequency & $5.405 \mathrm{GHz}$ \\
Slant-range pixel spacing & $4.73 \mathrm{~m}$ \\
Azimuth pixel spacing & $4.72 \mathrm{~m}$ \\
\hline
\end{tabular}

Two sets of five Radarsat-2 images each were acquired over this area; all of them with the same mode, beam and orbit pass, in order to build time series in the most consistent way. The list of available images (acquisition dates) and some basic information about these images are presented in Tables II and III, respectively.

The dates of acquisition of the images are illustrated in Fig. 2 together with the phenological evolution of the monitored parcels for both years. The phenological development of these parcels shows a clear common trend, but there are also important differences between parcels since some of them develop faster than others at some stages. During 2009 the available images cover the first part of the cultivation cycle, with one acquisition every 24 days (in fact, the first image is previous to all sowing dates so it will not be used in this work). Instead, during 2010 there are two images acquired at the early season, one at the end of the vegetative phase, and two more at the end of the growth cycle.

Following the same approach described in [9], a simple linear interpolator will be employed for deriving the phenology (in $\mathrm{BBCH}$ scale) at the dates of the radar acquisitions, since the acquisition dates are not coincident with the ground campaign dates. This assumption constitutes an uncertainty source because the temporal evolution of $\mathrm{BBCH}$ may not be linear in many cases. This can be especially relevant in some transitions between $\mathrm{BBCH}$ stages, since, by definition, a $\mathrm{BBCH}$ value is assigned to a parcel when more than the $50 \%$ of the plants belongs to that stage, despite there are other plants more advanced or delayed.

The diversity of phenological conditions at the same date (up to $20 \mathrm{BBCH}$ stages in some cases) and the availability of data from two different years have been exploited in this study to provide a more detailed evolution of the radar observables as a function of phenology, since each image provides information of different phenologies in correspondence with the phenological diversity among parcels. Unfortunately, there is no radar acquisition in 2009 and 2010 during the main reproductive phase 
TABLE I

DESCRIPTION OF THE MONITORED PARCELS

\begin{tabular}{ccccccc}
\hline \multicolumn{7}{c}{$\mathbf{2 0 0 9}$} \\
\hline Label & Surface (ha) & Sowing date (DoY) & Plants $/ \mathrm{m}^{2}$ & Panicles $/ \mathrm{m}^{2}$ & Harvest date (DoY) & Yield (kg/ha) \\
\hline A & 13.14 & 17-may (137) & 315 & 530 & 07-oct (280) & 8.949 \\
B & 12.47 & 15-may (135) & 350 & 600 & 29-sep (272) & 8.729 \\
C & 40.5 & 07-may (127) & 850 & 1300 & 25-sep (268) & 10.400 \\
D & 4.34 & 24-may (144) & 400 & 512 & 08-oct (281) & 10.060 \\
E & 17.26 & 15-may (135) & 450 & 580 & 14-oct (287) & 9.000 \\
\hline \multicolumn{7}{c}{$\mathbf{c 0 1 0}$} \\
\hline Label & Surface (ha) & Sowing date (DoY) & Plants $/ \mathrm{m}^{2}$ & Panicles/m & Harvest date (DoY) & Yield (kg/ha) \\
\hline A & 13.14 & 22-may (142) & 469 & 620 & 19-oct (292) & 9.493 \\
B & 12.47 & 22-may (142) & 464 & 510 & 18-oct (291) & 8.400 \\
C & 40.5 & 17-may (137) & 425 & 560 & 02-oct (275) & 10.057 \\
D & 4.34 & 23-may (143) & 350 & 640 & 13-oct (286) & 10.495 \\
E & 17.26 & 02-jun (153) & 183 & 549 & 04-nov (307) & 9.500 \\
F & 9.68 & 27-may (147) & 380 & 540 & 27-oct (300) & 9.403 \\
\hline
\end{tabular}

(BBCH from 50 to 70), so that interval will remain not observed and should be studied in future works.

\section{ANALYSIS OF POLARIMETRIC OBSERVATIONS}

\section{A. Basic processing and backscattering coefficients}

All images were coregistered by using the available orbital information. Then spatial averaging with a 9x9 boxcar filter was applied to estimate the polarimetric covariance matrices of every pixel in the images. Simultaneously, noise was estimated and removed from the data as explained in [14]. The importance of this removal will be shown later in the text. Attending to the specific configuration of these Radarsat- 2 images, the equivalent number of looks provided by such 9x9 filtering is around 48 , which is clearly enough for obtaining reliable estimates of all polarimetric observables in the subsequent analysis [15]. All polarimetric observables analysed in the rest of the text were extracted from these multi-looked covariance matrices. Note that reciprocity is assumed all along this study.

This study is centred on the areas with available ground measurements, so the regions of interest (ROI) of every parcel were defined by using a GIS database. Figure 3 presents a mosaic of the RGB images obtained for every parcel and every acquisition date using the Pauli basis. For convenience, the area outside the ROIs of the parcels is masked out. In addition, those acquisitions which cannot be analysed due to the lack of ground measurements (e.g. taken after the harvest date) were also removed from this image.

In Figure 3 we can observe that at the first acquisition of each year backscatter is very low for all channels, due to the flooded condition of the ground (it is actually a water surface) and the absence of vegetation, since they correspond to the early stages of the cultivation cycle. Then dominant colour in most images is pink as a result of the important double-bounce scattering. Only at the two last acquisitions in 2010, which correspond to the maturation stage, the RGB image changes to a light bluish colour, denoting backscattering dominated by a random volume.

In order to check the consistency of this dataset with respect to previous results in the literature, Figure 4 shows the evolution of backscattering coefficients at the linear channels (HH, VV and HV). They are represented as a function of the phenological stage. This type of figure will be used in this paper to analyse the dependence of any observable upon phenology. The average value and the standard deviation within each parcel are plotted to illustrate their behaviour against crop development and their local variability, respectively. These figures include all the data from the two campaigns, 2009 and 2010, without distinction.

The trends and absolute values plotted in Figure 4 agree with the well known response from rice at C-band, as it has been documented in the past with satellite data (ERS, Radarsat, Envisat), data from ground-based scatterometers, and simulated values obtained by electromagnetic models, despite in most cases the data were taken in different parts of the world and with different cultivation practices [1], [2], [3], [8]. All backscatter powers increase from very low values at the first stages to a maximum value reached after stage 20 (tillering start). The $\mathrm{HH}$ backscattering coefficient increases further up to a maximum around stage 40 , thanks to the increase in size of all plant elements and the dominance of the double-bounce scattering generated by the interaction between the stems and the underlying water surface. This double-bounce imposes a lower backscatter level at the VV channel which is also affected by an extinction stronger than at $\mathrm{HH}$ due to the vertical preferred orientation of most plants elements. This difference between the responses at $\mathrm{HH}$ and $\mathrm{VV}$ is illustrated clearly by its ratio, shown in Figure 4d, which has been successfully exploited for pre-operational rice mapping and monitoring with multi-temporal data [5]. Concerning the crosspolar echo, it starts from values lower than the copolar ones, but also exhibits a high increase driven by the emergence and development of the plants up to stage 20-30.

From stage 40 (booting) onwards the evolution of the backscattering coefficients is less pronounced than in the first part of the growth cycle. The HH channel decreases slowly, whereas the VV channel shows a variable minimum at stages 40 to 55, as a result of the important extinction at this polarisation, and then an increase to the end of the cycle. The crosspolar presents a moderate dip and then a recover to the end of the campaign. However, the interval between stages 55 and 80 is missing in the available Radarsat- 2 images, so this remains to be confirmed in the future.

As mentioned above, all these results based exclusively on the backscattering coefficients are not new and, indeed, constitute the core of most of the studies carried out so far with radar remote sensing applied to rice mapping and monitoring. 


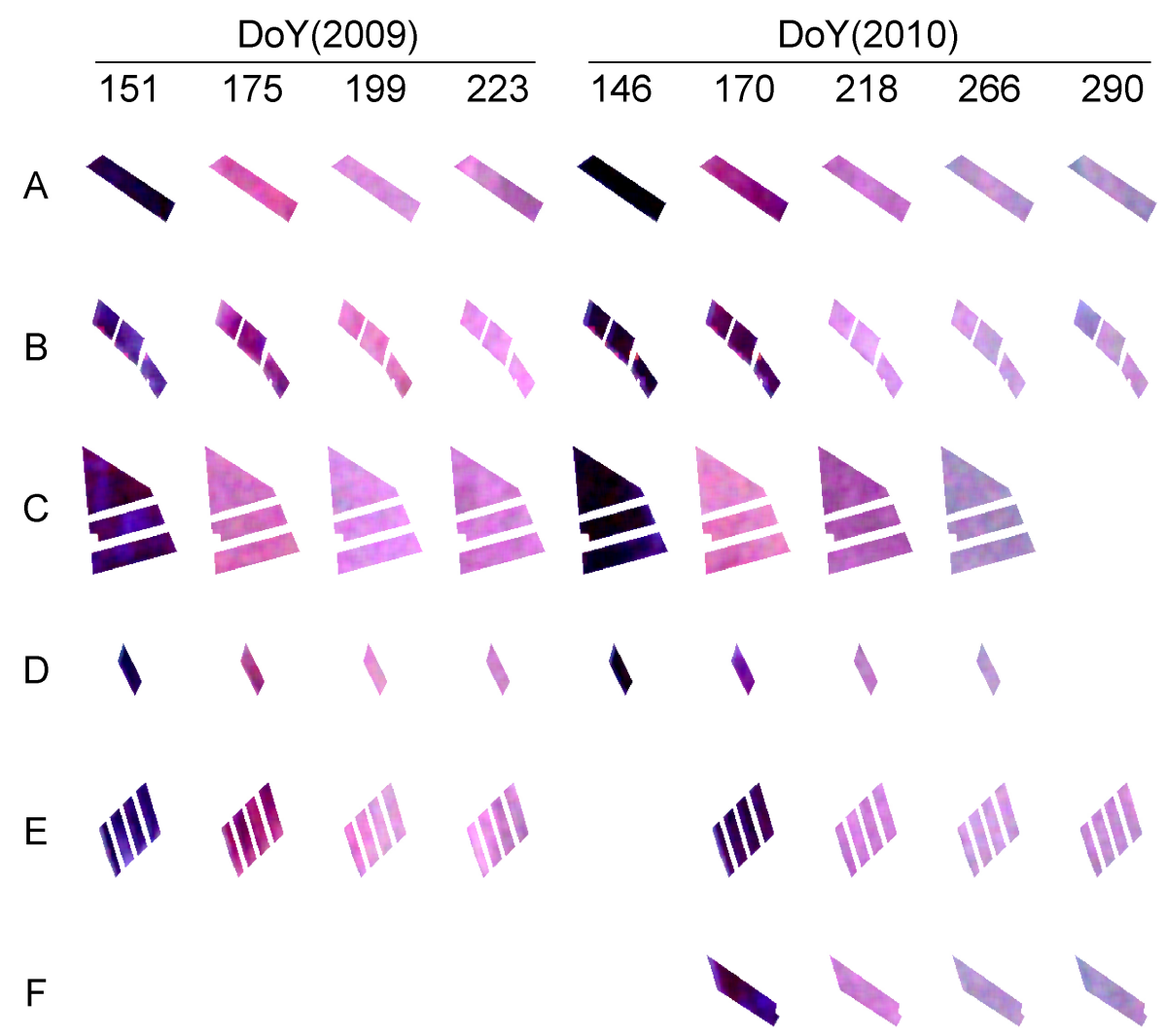

Fig. 3. Mosaic of the RGB composites obtained in the Pauli basis at all parcels locations and acquisition dates. Colour coding: Red $=\mathrm{HH}-\mathrm{VV}$, Green $=2 \mathrm{HV}$, and Blue $=\mathrm{HH}+\mathrm{VV}$. All channels are obtained as calibrated backscattering coefficients and mapped in the same range: Minimum $=-30 \mathrm{~dB}$, and Maximum $=-5 \mathrm{~dB}$. Values exceeding these limits are saturated to the extreme values. Images without corresponding ground measurements have been masked out.

They have been included here as a reference because the rest of the study is devoted to show the potential added value of full polarimetry when applied to rice monitoring and, specifically, to estimate phenology by exploiting a quad-pol acquisition at a particular date.

\section{B. Eigenvalue/vector decomposition}

To exploit the information contained in the fully polarimetric dataset provided by Radarsat- 2 we have employed in first instance the well known eigenvalue/vector decomposition proposed in [16], [17]. We used the same approach in [9] to analyse a TerraSAR-X dataset over the same test site, but in that case the observation space was restricted to a dual-pol coherent combination formed by $\mathrm{HH}$ and VV polarisations. Therefore, we can also check here the information added by full polarimetry with respect to dual polarimetry.

Figure 5 presents the evolution as a function of phenology of four parameters derived from such decomposition, namely: entropy $H$, anisotropy $A$, average alpha angle $\bar{\alpha}$, and dominant alpha angle $\alpha_{1}$.

In first place, we observe in Fig. 5a that entropy presents three different ranges during the cycle: moderate to high values $(0.5-$ 0.75) during the first stages (below stage 30), low to moderate values $(0.4-0.6)$ during stages 30 to 55 , and then high values $(0.6-0.85)$ at the maturation phase.

The absence of extremely high entropies along the crop devel- opment, but at the very late stages, indicates that the alpha angle parameter of the dominant eigenvector provides useful information about the most important scattering mechanism present at each phenological stage. Accordingly, the dominant alpha (see Fig. 5d) is mostly below 30 degrees at stages 0 to $18-20$ (tillering start), denoting that scattering from the water surface was the main contribution to the backscattered signal. Then, from that moment and until stage 30, i.e. during the whole tillering stage, the dominant alpha is well above 45 degrees which corresponds to dihedral scattering. This means that backscatter is dominated by the double-bounce interaction between the stem (and tillers) and the water surface. During the rest of the vegetative phase (stages 30 to 50) we can appreciate that the dominant alpha is consistently within a small interval around 45 degrees. This behaviour, together with a low entropy (0.4-0.6) indicates that the radar echo can be well described by a single scattering mechanism, which is a linearly polarised return. Such a response is due to both the double-bounce backscattering and the extreme extinction at vertical polarisation. The same observation was obtained for that period at X-band in [9]. Finally, at the last stages the dominant alpha angle is again below 45 degrees but separate from zero (not surface scattering). At this point it is interesting to compare dominant alpha (Fig. 5d) with the most commonly employed average alpha (Fig. 5c). The dynamic range of average alpha is much lower than the dominant alpha angle, due to the influence of the other mechanisms obtained by this de- 


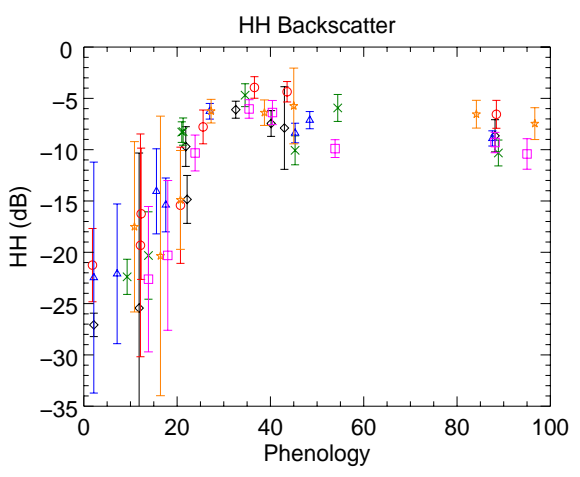

(a)

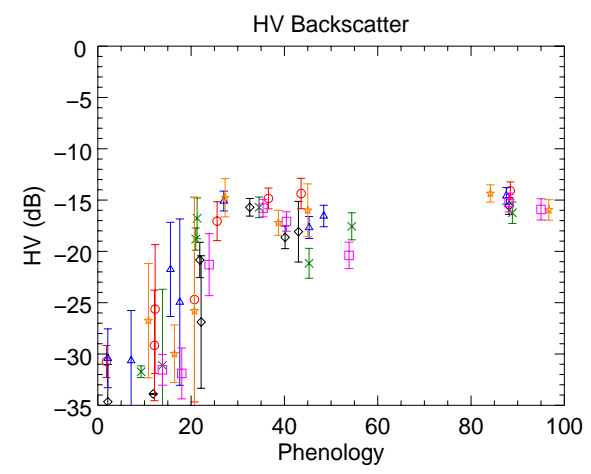

(c)

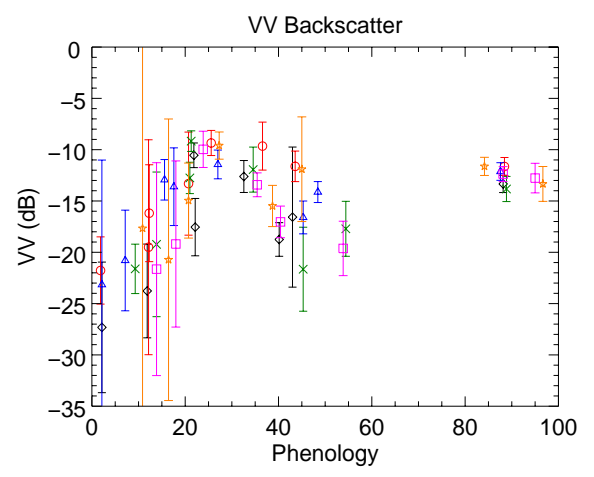

(b)

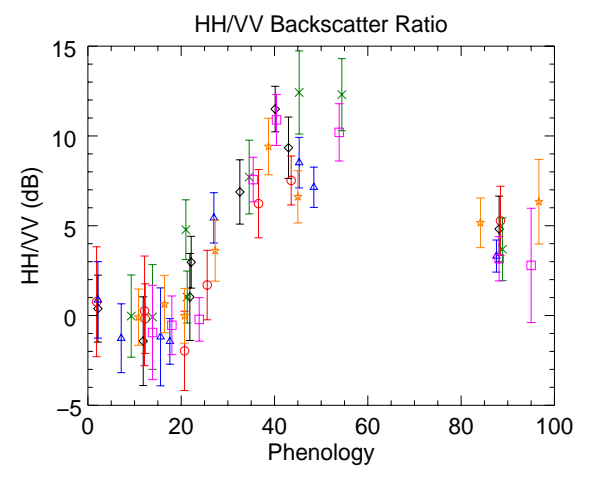

(d)

Fig. 4. Evolution of the backscattering coefficients at linear basis vs phenology for all monitored parcels. Averages and standard deviations are computed within each parcel. Parcels are denoted with different colours following the criterion used in Fig. 2. (a) HH, (b) VV, (c) HV, (d) HH/VV ratio.

composition, hence masking partially the behaviour described for each phenological stage.

A parameter presented in this analysis but not in [9] is anisotropy (see Fig. 5b), since it is not defined for dual-pol data. Anisotropy is quite high (above 0.5 ) during the initial stages (up to stage 30 approximately), and it remains uniformly around 0.2 to 0.4 for the rest of the campaign. Its physical interpretation, in terms of number of scattering mechanisms present in the scene is better explained jointly with entropy. From stage 0 to 30 we observe high anisotropy and moderate-to-high entropy, so there is a dominant scattering mechanism and a second non-negligible scattering component. The first dominant mechanism, indeed, changes from surface to double-bounce around stage 20, as described by the dominant alpha. From stage 30 to 50 the low entropy denotes a single scattering mechanism, for which anisotropy is not so relevant. Finally, at the maturation phase, high entropy and low anisotropy denote the presence of three scattering mechanisms (none of them negligible) or, based on the actual structure of plants at that stage, scattering from an almost random volume. Therefore, the role of polarimetry at this last stage is limited to certify such randomness.

Some additional comments are pertinent at this point to close the analysis of these observables. The first one is related to the noise removal applied to the data. The noise introduced by the Radarsat-2 system in the data is very low when compared to other satellite sensors. For the beam employed in this study, the noise equivalent sigma zero (NESZ) annotated in the product files is around $-35 \mathrm{~dB}$, much better than the $-19 \mathrm{~dB}$ present in TerraSAR-X. However, and recalling Fig. 4, the backscattering coefficients measured at the beginning of the cultivation cycle are close to that NESZ, or even below, especially for the crosspolar channel. Consequently, the estimation of polarimetric observables would be affected by noise during the first stages if it is not removed. For our purposes the main consequence of noise would be a moderate increase of entropy and a significant decrease of anisotropy, in both cases only for stages 0 to 20. As a result, the interpretation of anisotropy would be compromised.

It is worth reminding that in the study described in [9] we found that entropy showed extremely high values at the beginning of the tillering phase (around stage 20) and at the maturation phase (stages 80+). We postulated a different interpretation for both periods: the presence of two equally dominant scattering mechanisms at the tillering phase (e.g. surface scattering and double-bounce interaction) and random volume scattering at the end of the season. However, this explanation could not be demonstrated because only dual-pol data were available. Fortunately, with full polarimetry and exploiting both entropy and anisotropy this ambiguity has been solved as demonstrated in the previous paragraphs, at least at C-band.

In close relationship with this argument, it is also worth inspecting the entropy and dominant alpha parameters that would be obtained at C-band if the sensor were acquiring only $\mathrm{HH}$ and VV channels in a coherent way (same mode as TerraSAR-X). These two observables are represented in Fig. 6. The main difference in these plots with respect to the fully polarimetric case (shown in Fig. 5a and 5d) is an increased entropy in the dual- 


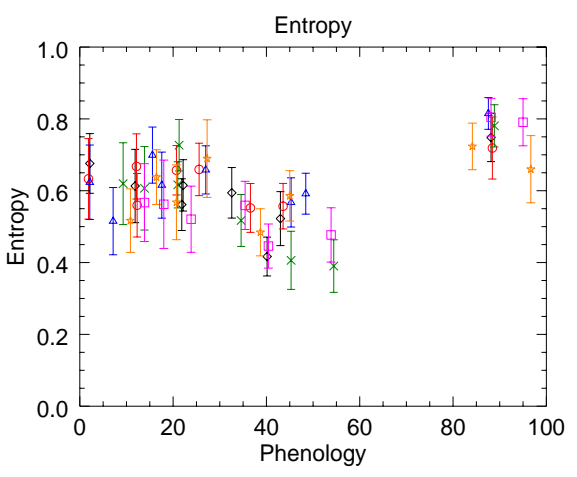

(a)

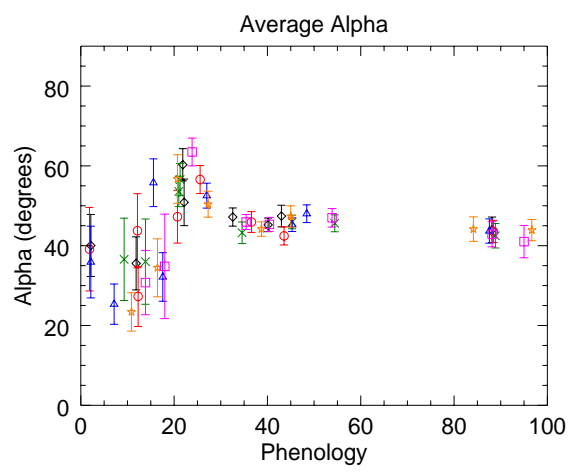

(c)

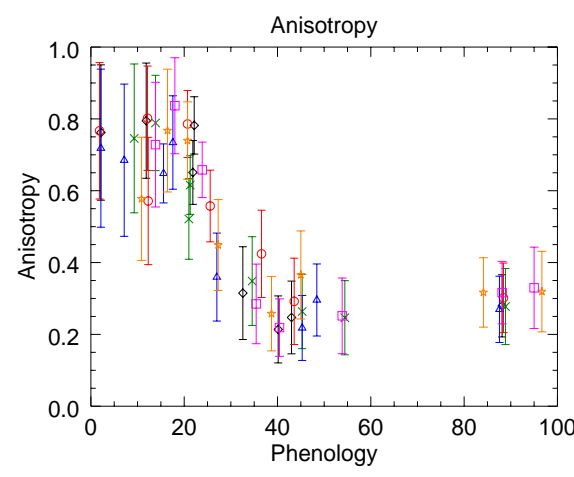

(b)

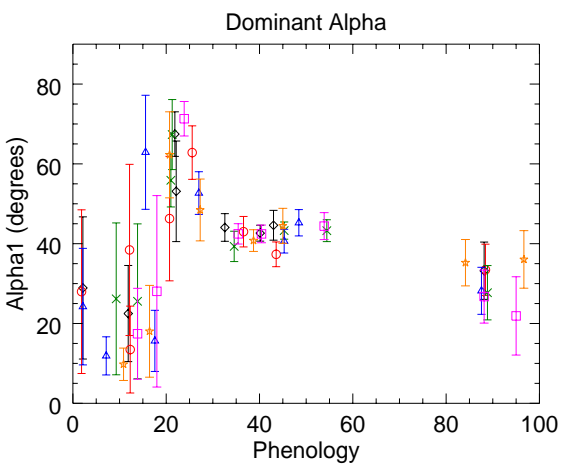

(d)

Fig. 5. Evolution of observables provided by the eigenvalue/vector decomposition of the coherency matrix vs phenology for all monitored parcels. Averages and standard deviations are computed within each parcel. Parcels are denoted with different colours following the criterion used in Fig. 2. (a) Entropy, (b) Anisotropy, (c) Average alpha angle, (d) Dominant alpha angle (alpha of the dominant scattering mechanism).

pol case at the early stages (until stage 30 ). This phenomenon is generated by the dimension of the observation space: 3 channels in full polarimetry and only 2 channels in dual-pol. Consequently, when there are only two scattering mechanisms in the scene, entropy is quite different in both cases. For instance, two equal eigenvalues $\lambda_{1}=\lambda_{2}=0.5$ produce a unit entropy in the dual-pol case, but if the third eigenvalue is null $\left(\lambda_{3}=0\right)$ entropy is just 0.63 in the quad-pol case. And this is just the case here, as it is confirmed also by the high anisotropy observed at the early stages. Regarding the dominant alpha angle, it is virtually the same in both configurations (see Fig.5d and Fig. 6b), hence confirming the usefulness of the HHVV dual-pol mode for inspecting the dominant scattering mechanism along the rice growth cycle.

If the C-band observations presented here are compared with the X-band ones analysed in [9], and provided that the incidence angles are quite similar (33 degrees for Radarsat-2 vs 30 degrees for TerraSAR-X), we conclude that most comments and physical interpretations of the retrieved data are valid for both frequency bands. Additional studies aimed at identifying the specific scattering physics for both bands at each stage (e.g. using scatterometres) would be useful to confirm this conclusion. A difference between both datasets, consequence of the wavelength, is the sensitivity to the start of the tillering stage. At $\mathrm{X}$-band the dominance of the double-bounce produced by the emergence of the first leaves and first tillers is firstly detected at stages 17-19, and only lasts until stages 21-22, giving way quickly to a linearly polarised backscattering characteristic of the vegetative phase. On the contrary, at C-band the transition to double-bounce dominance is detected around stage 20 , and it lasts until stage 30. The earlier response at X-band to the presence of plant elements is due to the bigger size of the leaves and tillers in terms of the wavelength. At X-band the radar is sensitive to stems and leaves around 15 to $20 \mathrm{~cm}$ long, whereas at Cband the radar becomes sensitive to 25 to $30 \mathrm{~cm}$ long elements. In parallel, the shorter duration at X-band of this phenological stage dominated by the double-bounce is due to the stronger extinction at higher frequencies. Therefore, in terms of sensitivity to phenology of rice fields, both bands are valid for monitoring purposes, but the transitions and the intervals with particular features may be shifted due to the intrinsic dependence of radar scattering and attenuation upon frequency. Clearly, the advantage of Radarsat- 2 over TerraSAR-X is the fully polarimetric configuration, which increases the observation space and, as already demonstrated, serves to solve some ambiguities present in the dual-pol case.

Finally, we have to mention that the lack of Radarsat-2 images during the reproductive stage (50-70) limits somehow the universality of this study. Anyway, from the experience gained with TerraSAR-X data and attending to the results obtained in [11], which include data at the reproductive stage, we can infer that this phenological interval is characterised by a smooth transition between the late vegetative and the maturation phase. Consequently, strange behaviours are not expected in the radar data at 


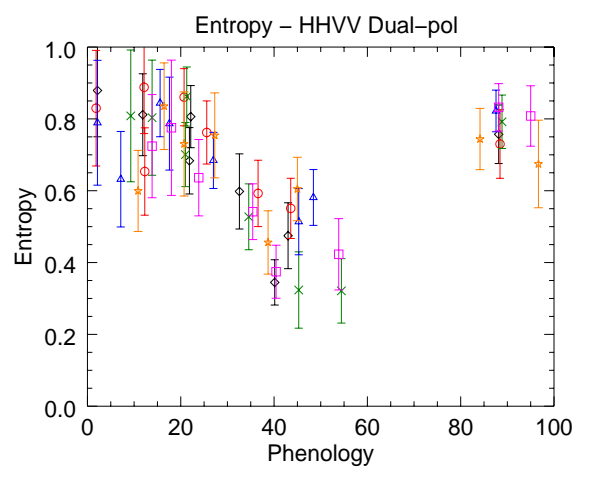

(a)

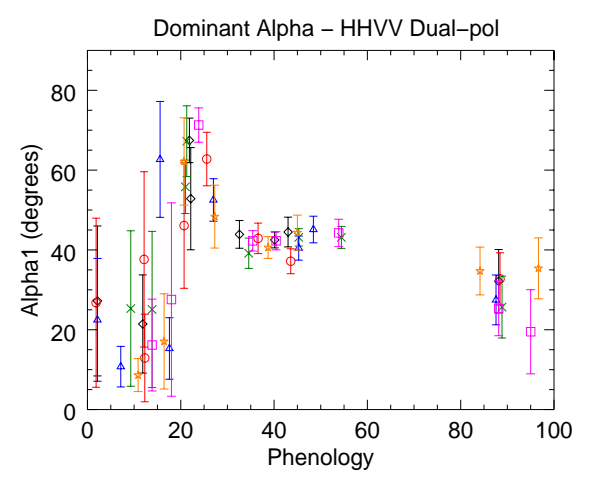

(b)

Fig. 6. Evolution of observables provided by the eigenvector/value decomposition of the HHVV dual-pol coherency matrix vs phenology for all monitored parcels. Averages and standard deviations are computed within each parcel. Parcels are denoted with different colours following the criterion used in Fig. 2. (a) Entropy, (b) Dominant $\alpha$ angle ( $\alpha$ of the dominant scattering mechanism)

that part of the growth cycle.

\section{Freeman-Durden decomposition}

A second widely used decomposition is applied also here to the Radarsat- 2 data on rice. It is a model-based decomposition proposed in [18] whose outputs are three backscattering contributions assigned to odd-bounce (surface) scattering, doublebounce (dihedral) scattering and volume scattering. A modified version avoiding non physical solutions (i.e. negative powers) [19] has been used [20]. Figure 7 presents the three contributions, and the ratio of the volume component over the sum of the other two, which can be interpreted as a volume-to-ground ratio.

This decomposition assigns the crosspolar channel to the volume scattering, hence the evolution shown in Fig. 7c. Regarding the other two terms, which are physically related with direct scattering from the underlying water surface and the doublebounce interaction between plants and water surface, they follow the expected behaviour according to the comments in previous sections. More precisely, surface scattering is stronger than dihedral scattering up to stage 20 , and then dihedral scattering dominates from stage 20 to 30 . From stage 30 to 50 there is no clear dominance, and eventually surface scattering is again over the dihedral contribution at stages $80+$. During the whole vegetative stage (0-50) the volume scattering is below the sum of the other two contributions (see Fig. 7d), but at the maturation phase they converge to similar values (being higher in some cases), as expected from a random volume.

\section{Compact-pol observables}

A known limitation of all satellite quad-pol systems to date, also shared by the HHVV coherent dual-pol mode of TerraSAR$\mathrm{X}$, is the reduced swath produced by the doubled pulse repetition frequency (PRF) employed to scan all combinations of transmitted/received polarisations. To avoid such a constraint, an alternative approach, usually named as compact or hybrid polarimetry (compact-pol or hybrid-pol), has been proposed [21]. In short, it consists in transmitting only a circular polarisation and receiving two orthogonal linear ones (e.g. horizontal and vertical), thus maintaining the PRF and the spatial coverage as in single-pol systems. Despite the dimensionality of the compactpol observation space is reduced with respect to full polarimetry, it has shown great potential and similar performance to quadpol in many applications, including agriculture monitoring and crop-type mapping [22], [23]. This mode is planned for the next generation of Radarsat systems, the Radarsat Constellation Mission (RCM), so it is worth inspecting its capabilities in the context of rice monitoring.

Due to the different nature of the compact-pol observations, a different set of observables has been defined in the literature [21], all of them with physical meanings somehow analogous to equivalent parameters in full polarimetry. Figure 8 shows the evolution as a function of phenology of four of these observables, which have been considered as relevant for the type of scene we are studying. They include the degree of polarisation $m$ (closely related to entropy), the relative phase between received fields $\delta$, and the circular polarisation ratio $\mu_{c}$. In addition, we have plotted in Fig. $8 \mathrm{~b}$ the dominant alpha angle $\alpha_{s}$ recently defined in [24].

As expected, the evolution of the degree of polarisation (Fig. 8a) resembles entropy (Fig. 5a), with three different ranges at three phenological intervals: $0.3-0.7$ from stage 0 to $30,0.6-$ 0.8 from stage 30 to 50 , and $0.3-0.6$ at stages $80+$. Note that low degrees of polarisation are equivalent high entropies and viceversa, so the physical explanation of such a behaviour is the same as for entropy. In second place, the dominant alpha angle in compact-pol ( $\alpha_{s}$, Fig. 8b) is perfectly coincident with the fullpol dominant alpha angle (Fig. 5d), hence providing exactly the same information for this scene along the whole phenological cycle.

The relative phase $\delta$ (Fig. 8c) is quite noisy in some parts of the phenological cycle, especially during the late vegetative phase (stages 30-50), since the vertically polarised return is very attenuated and, thus, such relative phase between received fields is meaningless. However, it remains consistently above 0 at stages 0-20 and 80+, and below -100 degrees during the tillering phase (stages 20-30). This values are in agreement with the dominant scattering mechanism (surface or double-bounce) for all these stages, which are characterised by different phase differences. Anyway, such behaviour is better described by the circular polarisation ratio $\mu_{c}$ (Fig. 8d), which resembles perfectly the dominant alpha angle but with a different scale: it is well below 1 (surface scattering) for stages 0-20, clearly above 1 (double-bounce scattering) at stages 20-30, consistently around 


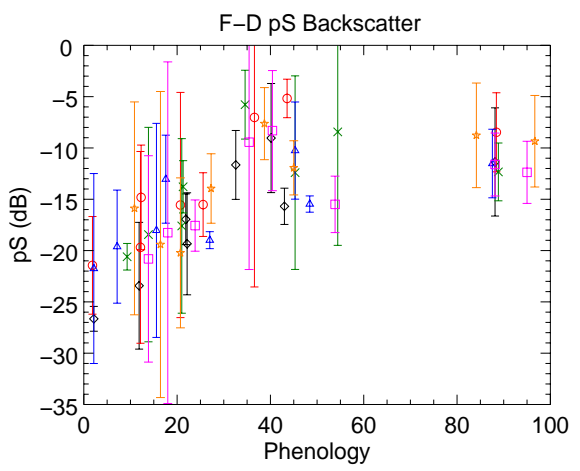

(a)

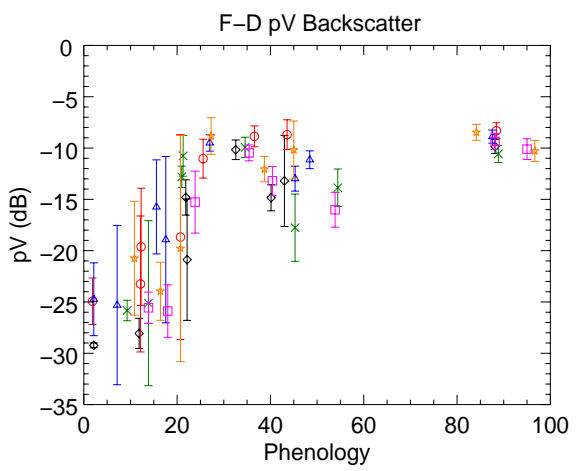

(c)

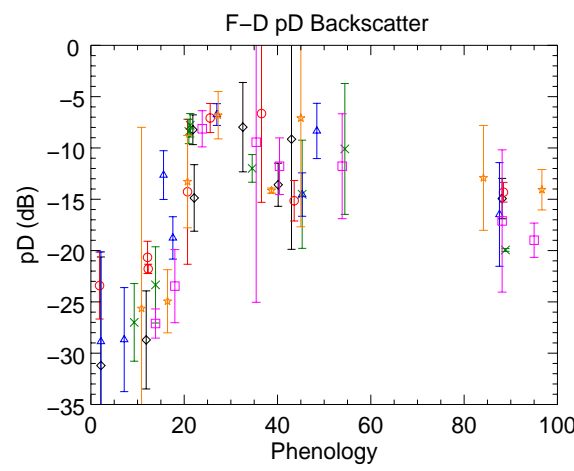

(b)

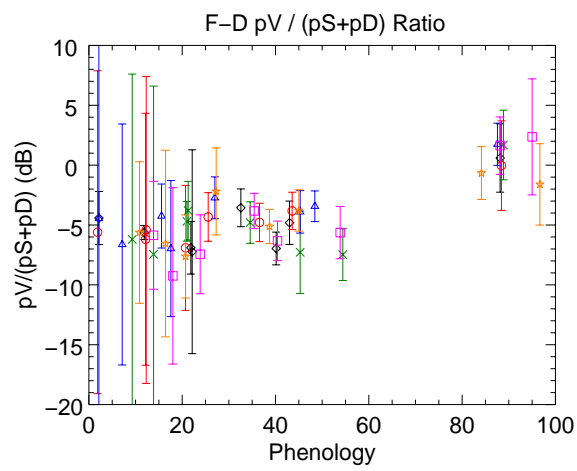

(d)

Fig. 7. Evolution of observables provided by the Freeman-Durden decomposition vs phenology for all monitored parcels. Averages and standard deviations are computed within each parcel. Parcels are denoted with different colours following the criterion used in Fig. 2. (a) Odd-bounce (surface) scattering, (b) Double-bounce (dihedral), (c) Volume scattering, (d) Ratio of volume to ground (surface + dihedral) scattering.

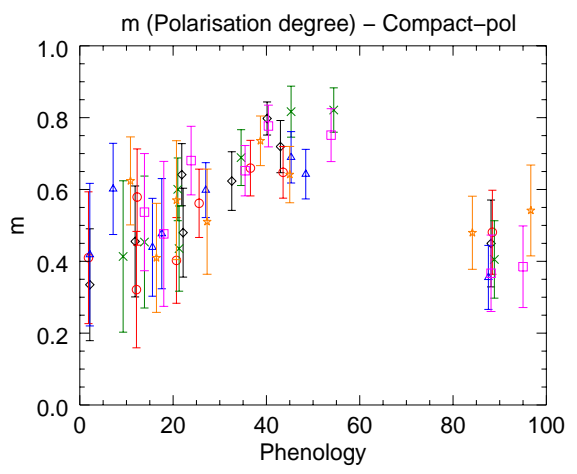

(a)

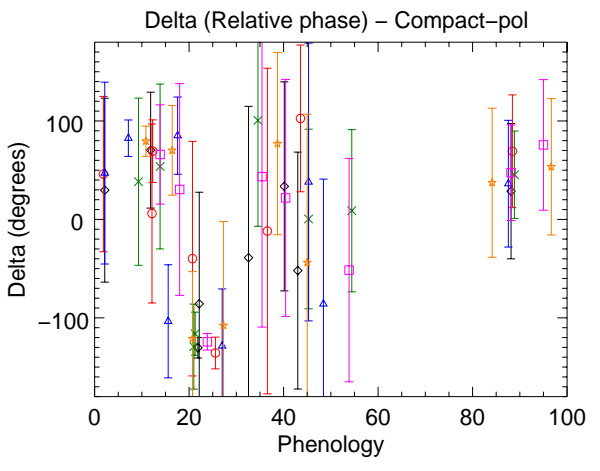

(c)

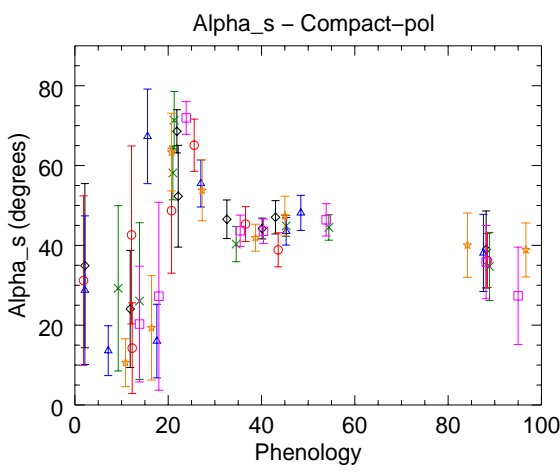

(b)

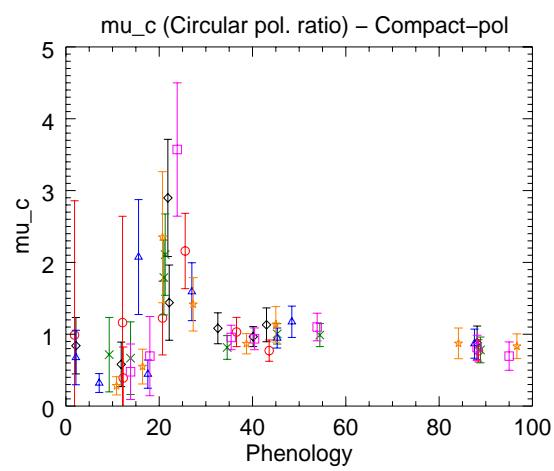

(d)

Fig. 8. Evolution of typical observables provided by a compact-pol configuration vs phenology for all monitored parcels. Averages and standard deviations are computed within each parcel. Parcels are denoted with different colours following the criterion used in Fig. 2. (a) Degree of polarisation $m$, (b) Dominant alpha angle $\alpha_{s}$, (c) Relative phase $\delta$, (d) Circular polarisation ratio $\mu_{c}$. 
1 (linear or dipole backscattering) at stages 30-50, and, eventually, slightly below 1 at the last stages of the cycle. In this sense, the information provided by both $\alpha_{s}$ and $\mu_{c}$ parameters is coincident. Finally, due to the reduced dimension of the observation space, there is no equivalent in this configuration to anisotropy, so the information contained in such an observable cannot be retrieved in compact-pol.

Figure 9 presents the last results of this analysis, which correspond to the outputs of the Freeman-Durden decomposition for compact-pol data, as defined in [24]. If they are compared with the same components retrieved in the full-pol case (Figure 7) we can appreciate both similarities and differences. For instance, the overall evolution of the volume contribution is almost the same in both compact-pol and full-pol, but the absolute level during the early stages (0-20) is higher in the compactpol case. Contrarily, the double-bounce contribution retrieved in compact-pol during the first stages is the lowest one among the three components, and lower than the values retrieved in the full-pol case. However, during the tillering phase (stages 2030 ), the double-bounce dominates over the other components as in the full-pol case. In addition, the ratio of the volume contribution over surface plus double-bounce is also different for both configurations at stages $0-30$, showing a decreasing trend in the compact-pol case and a constant level in full polarimetry. During the maturation phase, where polarimetry is not very important, both configurations provide the same observations for this decomposition.

\section{RETRIEVAL OF PHENOLOGY}

\section{A. Fully polarimetric data}

The analysis described in Section III allows us to conclude that the polarimetric signature of the rice fields under study are significantly different for different phenological stages. Consequently, the current phenological stage of a field under observation could be estimated by observing its polarimetric response. This idea is equivalently used in classification algorithms based on polarimetric data, e.g. for crop type mapping. In our case, however, we know the crop type (rice) and the different classes correspond to phenological intervals. The same approach was already tested in [9] with dual-pol HHVV TerraSAR-X data, providing successful estimates in most cases but also showing some ambiguities or errors that were justified by the reduced dimension of the observation space. In particular, stages around the tillering start (stage 20) were confounded with the maturation phase $(70+)$ in many instances, since both entail high entropies in this dual-pol mode. Therefore, this study will address such an issue to check whether full-polarimetry can solve that problem or not.

In order to maintain the algorithms and their physical rationales as simple as possible we apply the inversion to each Radarsat-2 acquisition independently, i.e. without exploiting any other temporal or auxiliary information different from the location of the rice fields. Moreover, we have chosen a set of polarimetric observables as reduced as possible.

From the previous analysis, the phenological intervals that can be identified by their polarimetric response in a straightforward way are the following:
1. Stages 0-20: Early vegetative phase (germination and leaf development). Radar backscatter is characterised by low (but increasing) levels at all channels, moderate-to-high entropy, dominant surface scattering (low alpha angle values), and high anisotropy.

2. Stages 20-30: Tillering. Radar backscatter is dominated by the double-bounce interaction between stems/tillers and the underlying water surface (alpha angle values above 45 degrees), with same range of entropies and anisotropy as in previous stages.

3. Stages 30-55: Advanced vegetative phase (stem elongation and booting). Radar echoes are linearly polarised (alpha angle close to 45 degrees), with low entropy as a result of the doublebounce and the extreme extinction at vertical polarisation.

4. Stages 55-80: Reproductive phase (heading and flowering) and early maturation (fruit development). Unfortunately, this phenological interval cannot be characterised in our study because there were not any Radarsat- 2 acquisitions at dates with such a phenology in the monitored fields.

5. Stages 80-100: Maturation phase (ripening and senescence). The nearly random volume structure of the plants produces high entropy and low anisotropy, while the dominant alpha angle falls below 45 degrees.

It is important noting that the second interval in this study at $\mathrm{C}$-band is shifted and stretched with respect to the equivalent interval for X-band employed in [9], which was restricted to stages 18 to 22 . The cause of this change, related with the size of all plant elements in terms of the wavelength, was already explained in Section III.

According to the definition of all these intervals and their physical description, a very simple algorithm for phenology retrieval can be designed based on three parameters: entropy, anisotropy and dominant alpha angle. The decision rules can be expressed as a hierarchical tree or as a graphical scheme with the corresponding ranges for each observable. This scheme is depicted in Fig. 10. The possible phenological intervals to be estimated are restricted to 1, 2, 3 and 5, since number 4 was not available as commented previously. Anyway, the numeration has been maintained for consistency with the results published in [9] and for future studies when, hopefully, data will be available for such an interval.

As it is denoted in Fig. 10, phenological intervals can be defined by one, two or three observables. For instance, the second interval (stages 20-30) can be identified by just observing the dominant alpha angle. On the other extreme, the maturation stage needs all three parameters to solve coincidences with other possible stages.

Despite other radar observables could be used to identify some of the phenological stages, we prefer this small set of polarimetric descriptors which are not dependent on absolute backscattered powers and that provide a clear explanation on the scattering phenomena present in the scene at every stage. For completeness, Table IV presents a list of parameters that could be used alternatively to identify these phenological stages, despite some of them were not included in the previous analysis due to space constraints. In general, observables derived from decompositions provide better physical explanations and are less dependent on particular features of the fields, absolute 


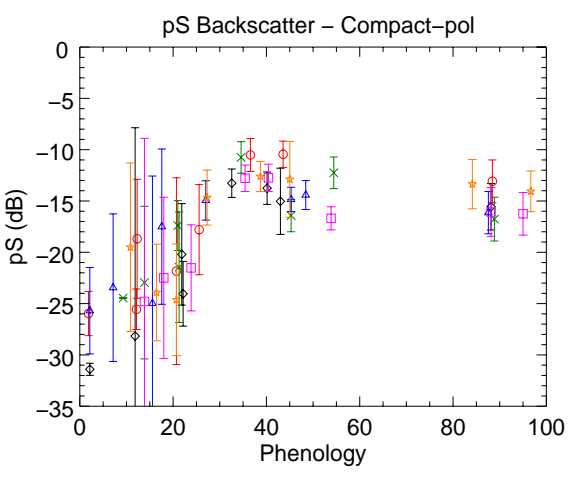

(a)

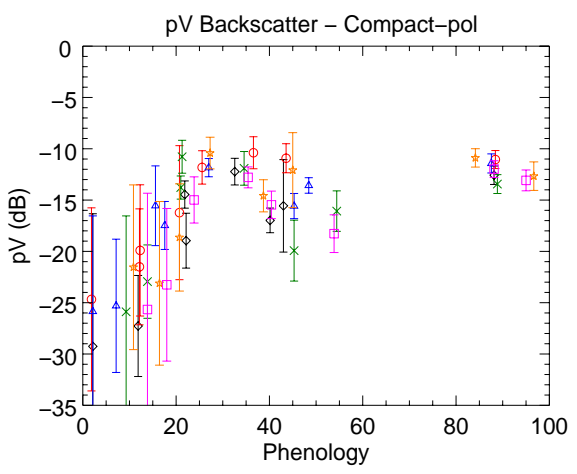

(c)

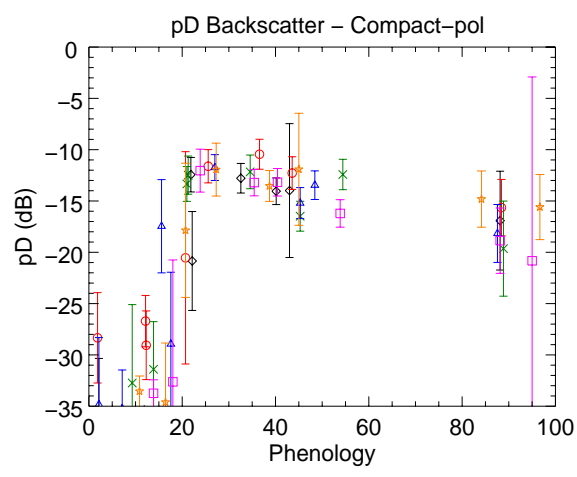

(b)

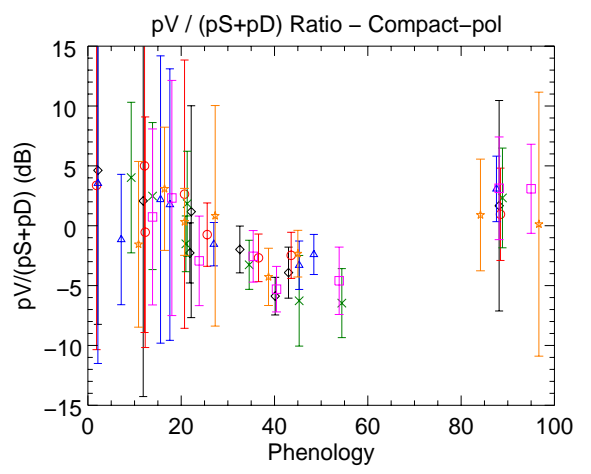

(d)

Fig. 9. Evolution of observables provided by the Freeman-Durden decomposition of compact-pol data vs phenology for all monitored parcels. Averages and standard deviations are computed within each parcel. Parcels are denoted with different colours following the criterion used in Fig. 2. (a) Odd-bounce (surface) scattering, (b) Double-bounce (dihedral), (c) Volume scattering, (d) Ratio of volume to ground (surface + dihedral) scattering.

calibration issues, incidence angles, etc. Therefore, the definition of thresholds and classification rules is easier and less arbitrary than in the case of backscattering coefficients. Moreover, as shown in [11] with an experiment related to rice mapping with Radarsat-2, decomposition parameters provide better results than backscattering coefficients, probably due to saturation effects (according to the analysis provided by authors in [11]) which were observed in the power of the radar echoes at advanced stages (see Fig. 4) but not in phase differences and correlations between channels.

Regarding the algorithm itself, the classification based on ranges of parameters is not exhaustive, since there are values and combinations of observables which are not assigned to any class (phenological stage). We also have to mention that many other algorithms could be applied, but this simple one provides very good results as it is illustrated in the following.

The output of this algorithm when applied to all (multilooked) pixels of the available images is shown in Fig. 11, where the arrangement of parcels and acquisitions is the same as in the mosaic shown in Fig. 3. We can appreciate that in most cases a single colour dominates whole parcels, which means that the retrieval algorithm assigns one phenological stage to the whole field homogeneously. In some situations, however, there appear large areas of mixed stages at the same field, in most cases corresponding to adjacent stages in the phenological cycle. This mixture of responses within a parcel is expected since plants usually develop at different rates in different zones of a field, es- pecially if the field is large and/or close to disturbing elements typical of the border of cultivation sites (highways, mountains, etc.). The detection of heterogeneous areas, as provided by this algorithm, can be useful to farmers and managers to verify the right response of the crops to cultivation practices (e.g. germination, fertilisation, etc.). Note that the total number of pixels not assigned to any class is quite low. Finally, we can also observe some discrepancies in the edges of the parcels, which in fact can be detected in the RGB mosaic. They are due to a small displacement of the available ROIs of the parcels with respect to their actual geographical location, hence including the response of paths and small walls inside the ROIs.

The validation of these results has to be carried out at parcel level, since the available ground data are expressed at that level: a single phenological stage (in the full $\mathrm{BBCH}$ scale) for each parcel and date. Consequently, the comparison of the ground data against the retrieval results is performed by first computing the number or percentage of pixels assigned to each phenological stage at each parcel, and then comparing the mode (most retrieved value) within each parcel with the ground data. This numerical validation is shown in Table V, which is composed of six sub-tables referred to all parcels. Each row in these sub-tables corresponds to a different Radarsat- 2 acquisition. For each parcel, the left side of the sub-table presents the number of pixels assigned to each class and also the number of unassigned pixels. The right part of the sub-tables shows the percentage of pixels assigned to each phenological stage, computed over the 
TABLE IV

LIST OF ALTERNATIVE OBSERVABLES USEFUL TO IDENTIFY EACH PHENOLOGICAL INTERVAL

\begin{tabular}{cl}
\hline Phenological interval & Observables \\
\hline $0-20$ & Low backscatter power at all channels \\
& HH/VV around $0 \mathrm{~dB}$ \\
& Moderate to high correlation between $\mathrm{HH}$ and $\mathrm{VV}$ \\
& Phase difference between $\mathrm{HH}$ and $\mathrm{VV} \approx 0$ \\
& Freeman-Durden: Ratio of volume to ground scattering around $-5 \mathrm{~dB}$ \\
& Freeman-Durden: Odd-bounce $>$ double-bounce (more than $5 \mathrm{~dB})$ \\
\hline $20-30$ & Phase difference between HH and VV below -90 degrees \\
& Freeman-Durden: Double-bounce $>$ odd-bounce (more than $5 \mathrm{~dB})$ \\
\hline $30-55$ & HH/VV above $6 \mathrm{~dB}$ \\
& High correlation between the first two Pauli channels \\
\hline $80-100$ & Freeman-Durden: Ratio of volume to ground scattering around or above $0 \mathrm{~dB}$ \\
\hline
\end{tabular}

$\operatorname{DoY}(2009)$

$151 \quad 175 \quad 199 \quad 223$

A

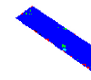

B
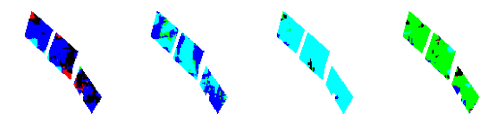

C
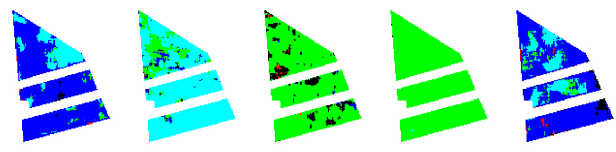

D
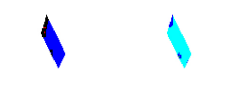

\section{$E$}
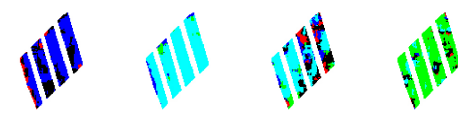

F
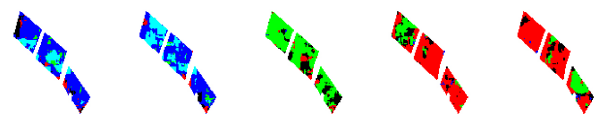

\begin{tabular}{cccc}
\multicolumn{4}{c}{$\operatorname{DoY}(2010)$} \\
\hline $146 \quad 170 \quad 218 \quad 266$ & 290
\end{tabular}
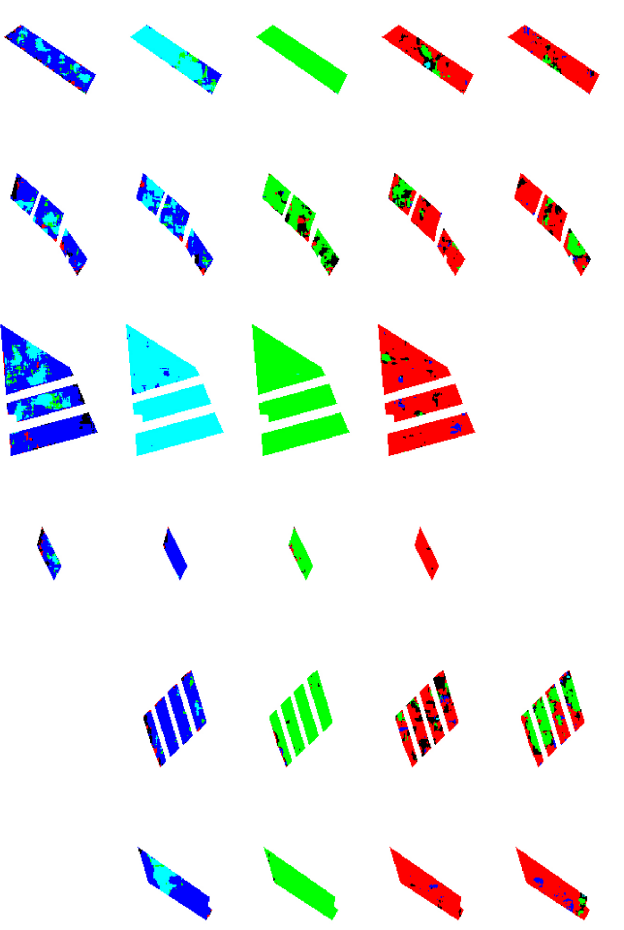

Fig. 11. Mosaic with the retrieval result for the fully polarimetric case. The colour code is the same used in Fig. 10. Black pixels were not assigned to any phenological stage.

total number of assigned pixels (i.e. discarding the unassigned ones). To ease the reading of this table, the font type of largest percentage, that defines the mode, is boldface and coloured with the colour assigned to its corresponding phenological interval $(1,2,3$, or 5). Finally, at the right extreme of the tables, the phenology value at each radar acquisition date, as provided by the ground campaign, is shown. In this case, the colour of the font used in the table also indicates the phenological interval. Therefore, to check whether the estimates agree with the ground data or not, one just has to check whether the colour of the mode is the same as the colour of the ground data.

Attending to the results presented in Table $\mathrm{V}$, the retrieval of phenology is correct, when evaluated at parcel level, in 44 of the 46 cases, i.e. in $96 \%$ of the cases, despite the simplicity of the approach. In most cases the percentage of the mode is above $70 \%$, with many cases above $90 \%$. There are several cases with lower percentages (e.g. parcel B at Doy 175 of 2009) which reflect that the phenological stage of the parcel is just at a transition between the defined intervals (e.g. stage 21 in this example), thus such a mixture of estimates between adjacent intervals is expected.

The two wrong estimates are marked with asterisks at the rightmost side of the tables. The first case (denoted with one asterisk) corresponds to Parcel D on DoY 175 of 2009. The value annotated in the ground campaign was stage 16 (taken the day after the radar image), whereas the algorithm retrieves the 
TABLE V

STATISTICS OF THE RETRIEVAL RESULTS FOR THE FULLY POLARIMETRIC CASE.

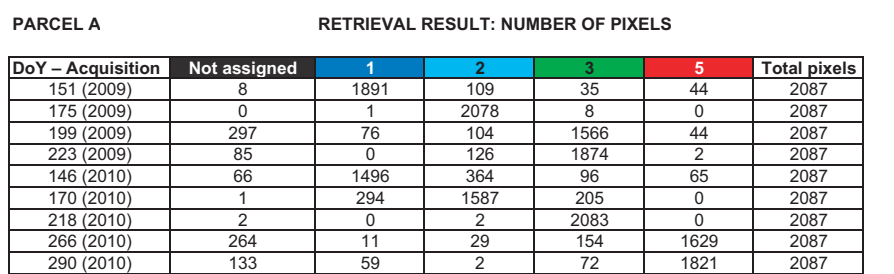

PARCEL B

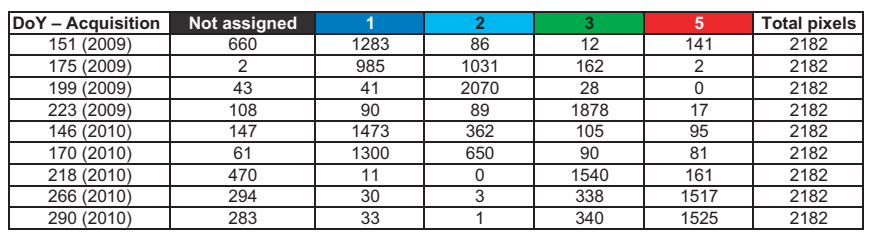

PARCEL C

RETRIEVAL RESULT: NUMBER OF PIXELS

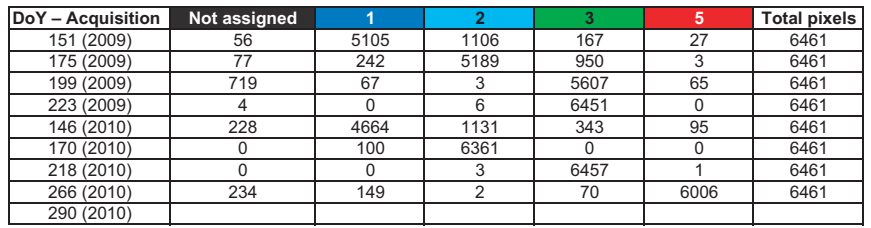

PARCEL D

RETRIEVAL RESULT: NUMBER OF PIXELS

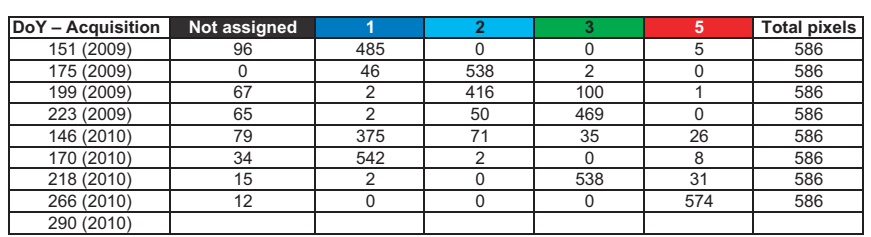

PARCEL E

RETRIEVAL RESULT: NUMBER OF PIXELS

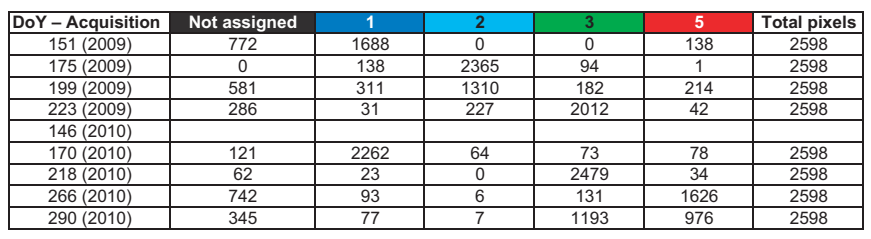

PARCEL F

RETRIEVAL RESULT: NUMBER OF PIXELS

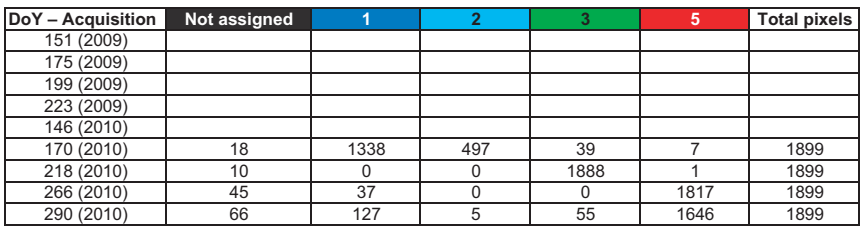

PERCENTAGE OVER ASSIGNED PIXELS



PERCENTAGE OVER ASSIGNED PIXELS

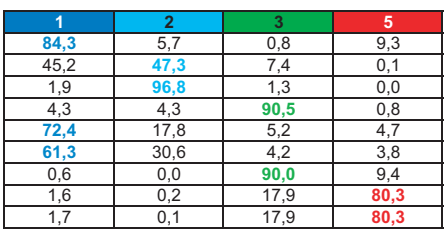

PERCENTAGE OVER ASSIGNED PIXELS



PERCENTAGE OVER ASSIGNED PIXELS

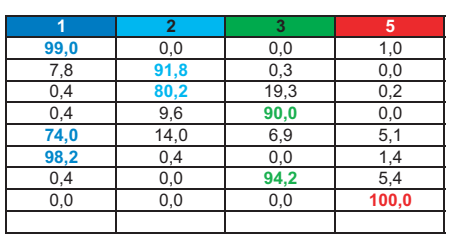

PERCENTAGE OVER ASSIGNED PIXELS



PERCENTAGE OVER ASSIGNED PIXELS

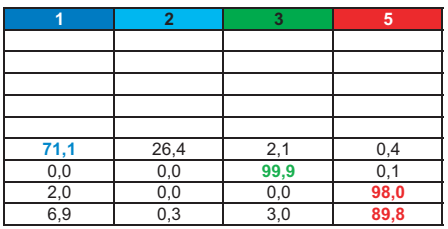

GROUND DATA



GROUND DATA

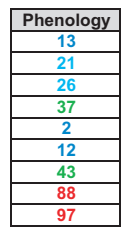

GROUND DATA

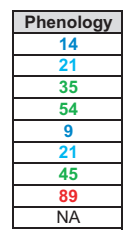

GROUND DATA

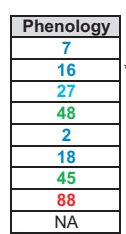

GROUND DATA



GROUND DATA

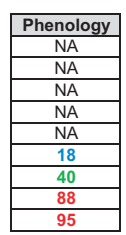

second interval (stages 20-30) with $92 \%$ of pixels assigned to that range. Table I and Figure 2 indicate that this parcel was the last to be planted in 2009, and at that date its development was still far from the rest of parcels (already at stages 21-22), respectively. However, when looking at the RGB mosaic in Fig. 3 we can appreciate that the aspect of this parcel (colour) is the same as the rest of parcels at that date. This parcel is represented by a blue triangle in Figs. 4 and 5, being the third from the left the point which corresponds to this date. We can appreciate how its values are in the same range as other parcels in stages 21-22. Specifically, its dominant alpha angle (easily visible Fig. 5d) presents a mean of more than 60 degrees, hence the retrieved phenology. It is important to notice that in the successive date of ground campaign (DoY 183) the annotated phenology was the same as for the rest of parcels, so this field exhibited a faster development at this period, which may explain a possible error in the ground campaign value. The second error (denoted with two asterisks) corresponds to Parcel E on the last date (DoY 290 in 2010). In this case, the ground campaign indicates that the parcel is in the last stages of the cultivation cycle, whereas the retrieval algorithm provides only $43 \%$ of pixels at maturation and $53 \%$ of pixels assigned to the advanced vegetative phase (stages 30-50). By looking either at the RGB mosaic (Fig. 3) or at the radar observables (last orange asterisk in Figs. 4 and 5) one 

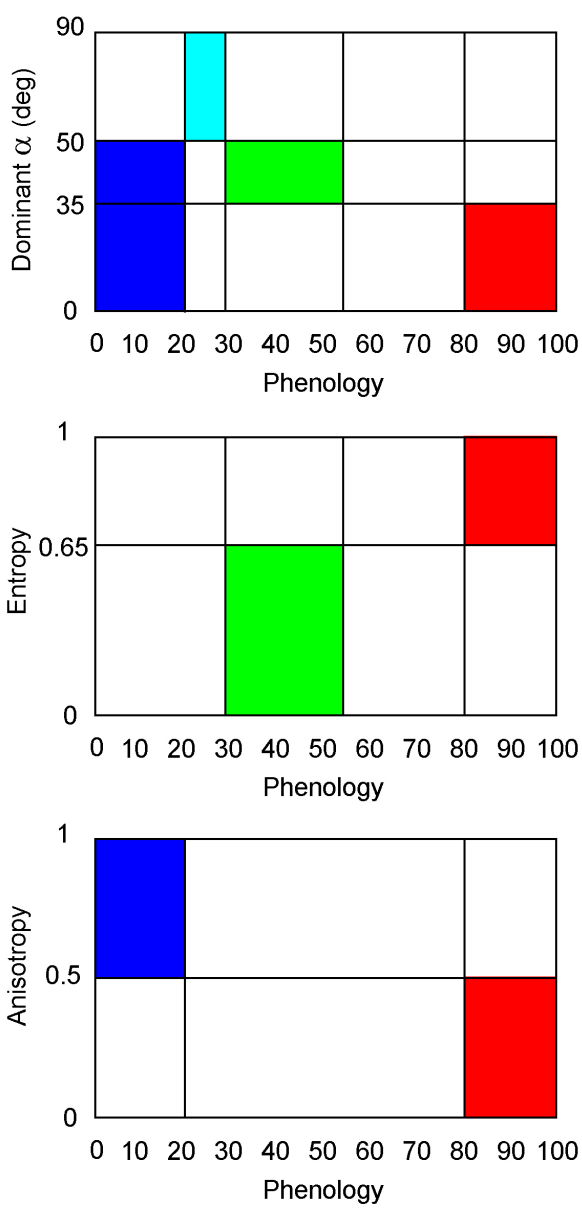

Fig. 10. Ranges employed in the phenology retrieval algorithm designed for fully polarimetric data. Observables: dominant alpha angle (top), entropy (centre) and anisotropy (bottom). Colour coding: Dark blue = Early vegetative (stages 0-20), Light blue: Tillering (stages 20-30), Green: Advanced vegetative (stages 30-55), Red: Maturation (stages 80-100). White areas are not used by the algorithm.

can confirm that the radar response of this parcel lies between both cases, and hence the divided assignation and the error when validating against the mode.

\section{B. Compact-pol data}

Once we have demonstrated the potential of full polarimetry at C-band for retrieving the phenology of rice fields (from a set of four possible intervals), it is pertinent checking the performance of compact polarimetry in the same application, provided the advantages of this configuration as it was mentioned in Section III.

Following the approach employed for full polarimetry we should select firstly a set of observables with a response to phenology similar to dominant alpha angle, entropy, and anisotropy, but defined for compact polarimetry. For the first two parameters the answer is evident: the compact-pol dominant alpha angle (denoted as $\alpha_{s}$ ) and the degree of polarisation $(m)$ can do the job, in agreement with the results and comments included in Section III. Concerning anisotropy, however, there is not any observable in the compact-pol domain with the same physical interpretation. Anyway, the role of anisotropy in the retrieval algorithm sketched in Fig. 10 is serving to distinguish early stages
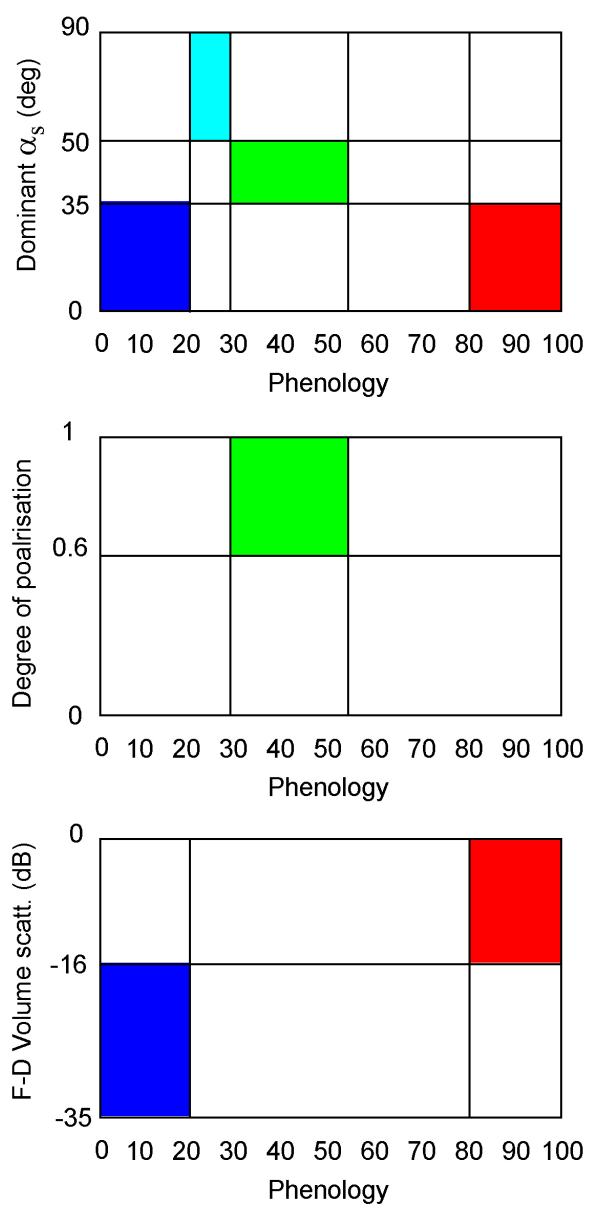

Fig. 12. Ranges employed in the phenology retrieval algorithm designed for compact-pol data. Observables: dominant alpha angle (top), degree of polarisation (centre) and volume component of the Freeman-Durden decomposition (bottom). The colour code is the same used in Fig. 10.

(0-20) and late stages (80+) which are both characterised by low alpha angles. Therefore, we should search for an observable with clearly different values at these two phenological intervals. Observing Figs. 8 and 9, we could use the volume or the doublebounce contribution provided by the Freeman-Durden decomposition. They exhibit low values at the early stages and high values at the end of the cycle.

Figure 12 illustrates the ranges of values for the retrieval scheme proposed here with compact-pol data, where the volume component has been employed by using a threshold of $-16 \mathrm{~dB}$. Again, it is a quite simple approach, in line with the goal of this study.

The results provided by this algorithm are shown in Fig. 13, and the corresponding validation statistics are included in Table VI. The general aspect of the retrieved phenology map is quite similar to that shown in Fig. 11 for fully polarimetric data, but there are more unassigned pixels than in that case. Anyway, the validation with the mode within each parcel provides the same overall success rate as with full-polarimetry: 44 of the 46 cases are assigned correctly to their phenological interval, being the same two cases that produced wrong results with fullpol data. A closer inspection to Table VI reveals that now it is more frequent obtaining lower percentages of pixels correspond- 
ing to the modes. In other words, there are higher percentages of wrongly assigned pixels (see for instance the red spots in Parcel C at DoY 199 in 2009, or in Parcel B at DoY 218 in 2010), but they are not enough to corrupt the average response at field level. Therefore, we conclude that compact polarimetry provided the same performance as full polarimetry for phenology retrieval at parcel level.

\section{Discussion about dual-pol data}

In order to provide the same spatial coverage (swath) as single-pol radar sensors, some satellites have been designed with an operational dual-polarimetric mode, as the future Sentinel-1a and 1b of the European Space Agency. In this case dual polarimetry consists in transmitting a linearly polarised wave and receiving simultaneously the like and the orthogonal polarisations. Therefore, the measured polarimetric channels are $\mathrm{HH}$ and $\mathrm{HV}$, or $\mathrm{VV}$ and $\mathrm{VH}$, i.e. a copolar channel and the crosspolar one ${ }^{1}$. The radar response from all agricultural scenes exhibit the so-called reflection symmetry [16] which makes the correlation between copolar and crosspolar channels to be null in theory (and close to zero in practice). As a result, the $2 \times 2$ covariance matrix that could be formed for each dual-pol data is a diagonal matrix, defined only by the real entries of its diagonal. Therefore, the only two observables provided by such systems are the backscattering coefficients of the copolar and the crosspolar channel.

For the rice phenology retrieval application we are studying here, the consequence of reflection symmetry is that we are restricted to use the crosspolar backscattering coefficient (Fig. 4c) and one of the two copolar coefficients $(\mathrm{HH}$ in Fig. $4 \mathrm{a}$ or VV in Fig. 4b) to define the retrieval algorithm. From these plots, it is evident that we could identify the first phenological interval (stages 0-20) thanks to its low backscatter at all channels. However, the rest of phenological stages do not present a clear separation, thus making impossible to approach that problem in the same way. As a potential alternative, in line with the design of the Sentinel-1 system, a multi-temporal approach could be designed, helped by the short revisit time of such a system (12 days for one satellite and 6 days for the pair). Anyway, this is out of the scope of this study.

\section{CONCLUSIONS}

This study has demonstrated that a C-band radar satellite with polarimetric capabilities can estimate the phenological stage of rice fields, from a set of four relevant intervals, by using just one acquisition without any other auxiliary information. This ability is based on the polarimetric response of rice fields, which can be clearly separated among different behaviours along their cultivation cycle. Consequently, a simple classification algorithm based on three observables can be used for retrieving phenology. The validation carried out at parcel level was successful for 44 of the 46 cases (96\%). In addition, the requirement of a fully polarimetric system can be alleviated by using a compactpol mode, with the same spatial coverage as single-pol systems, since the validation at parcel level produced the same results.

${ }^{1}$ This should be confounded with the copolar-copolar combination (HH and $\mathrm{VV}$ ) provided by TerraSAR-X, because in that case the swath is the same as with full polarimetry.
Despite the experiment was conducted in a temperate region and that rice types and cultivation practices differ importantly all over the world, the main findings of this work could be extrapolated to other sites, since they are based on basic scattering responses which are common to all rice fields. Anyway, the influence of particular aspects, like the absence of a water layer at some stages or the regular plantation grid generated by machines in some sites, should be studied in the future.

An obvious limitation of the present study is the lack of radar data during the reproductive stages (55-70) and early maturation (70-80). From our previous experience with TerraSAR$\mathrm{X}$ data [9] and recent results presented by other authors with Radarsat-2 images acquired during these late stages [11], the expected response from the fields during the reproductive phase is a rather smooth transition, for all observables, between the vegetative and the maturation phase. Therefore, we should refine the retrieval algorithm to include such an intermediate phenological interval. If the separation between stages is not very clear we can anticipate that some errors will be obtained around the two borders (stage 50 and stage 70), but it has to be confirmed with real data.

From the point of view of the final application of this approach by the end-users, it is evident that a short refresh time is required in the phenological information to help their cultivation practices, being one week a good sampling period. Since Radarsat- 2 is designed with a revisit time (in the same mode/beam) of 24 days, multiple beams (incidence angles) and passes (ascending/descending) should be combined to fulfil this requirement. In that case, the influence of incidence angle should be also assessed.

Future studies should include also radar images acquired at Lband, for instance with the next ALOS-2 satellite. In principle, at lower frequencies we expect to be less sensitive to the initial emergence of the plants, since they have to be taller to become visible to the radar (as we already experience when passing from $\mathrm{X}$ to C-band). On the other hand, once the rice plants are clearly developed (from stage 30 onward), the increased penetration capability of L-band may help to discriminate better among advanced stages.

\section{ACKNOWLEDGEMENTS}

The authors would like to thank the support of Manuel Cano, Fernando Carrascal and Santiago Aparicio, all from the Federacion de Arroceros de Sevilla, for providing the ground measurement data and for their helpful comments.

All RADARSAT-2 images were provided by MDA and CSA in the framework of project SOAR 2125. RADARSAT-2 Data and Products (C)MacDonald, Dettwiler and Associates Ltd. (2009-2010) - All Rights Reserved. RADARSAT is an official trademark of the Canadian Space Agency.

\section{REFERENCES}

[1] T. Kurosu, M. Fujita, and K. Chiba, "Monitoring of rice crop growth from space using the ERS-1 C-band SAR," IEEE Trans. Geosci. Remote Sensing, vol. 33, no. 4, pp. 1092-1096, July 1995.

[2] T. Le Toan, F. Ribbes, L.-F. Wang, N. Floury, K.-H. Ding, J. A. Kong, M. Fujita, and T. Kurosu, "Rice crop mapping and monitoring using ERS1 data based on experiment and modeling results," IEEE Trans. Geosci. Remote Sensing, vol. 35, no. 1, pp. 41-56, Jan. 1997. 


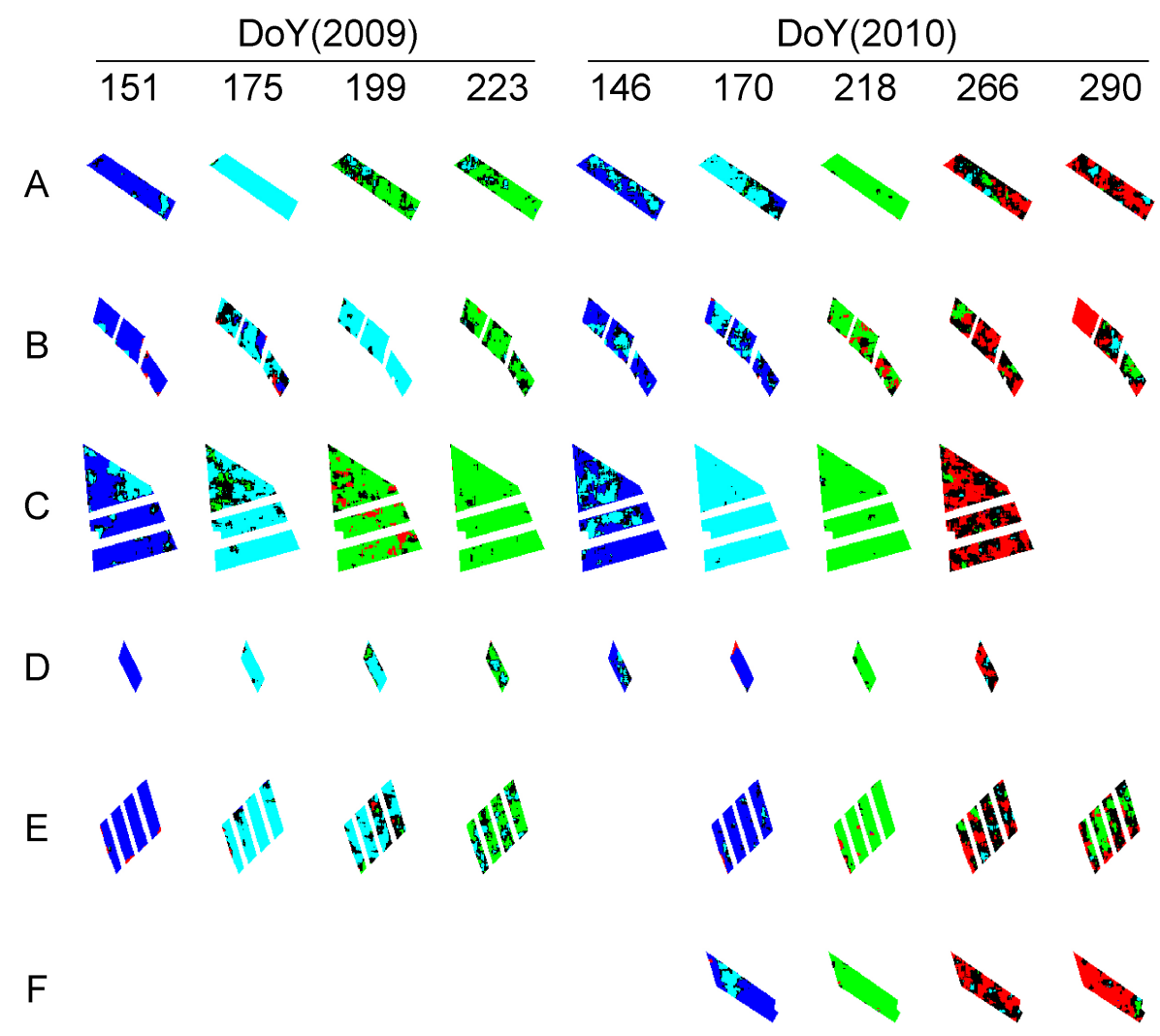

Fig. 13. Mosaic with the retrieval result for the compact-pol case. The colour code is the same used in Fig. 10. Black pixels were not assigned to any phenological stage.

[3] J.-Y. Koay et al., "Paddy fields as electrically dense media: Theoretical modeling and measurement comparisons," IEEE Trans. Geosci. Remote Sensing, vol. 45, no. 9, pp. 2837-2849, Sept. 2007.

[4] A. Rosenqvist, "Temporal and spatial characteristics of irrigated rice in JERS-1 L-band SAR,” Int. J. Remote Sensing, vol. 20, pp. 1567-1587, 1999.

[5] A. Bouvet, T. Le Toan, and N. Lam-Dao, "Monitoring of the rice cropping system in the Mekong delta using ENVISAT/ASAR dual polarization data," IEEE Trans. Geosci. Remote Sensing, vol. 47, no. 2, pp. 517-526, Feb. 2009.

[6] J. M. Lopez-Sanchez, J. D. Ballester-Berman, and I. Hajnsek, "First results of rice monitoring practices in Spain by means of time series of TerraSAR$\mathrm{X}$ dual-pol images," IEEE J. Selected Topics in Applied Earth Observations and Remote Sensing, vol. 4, no. 2, pp. 412-422, June 2011.

[7] T. Le Toan, H. Laur, E. Mougin, and A. Lopes, "Multitemporal and dualpolarization observations of agricultural vegetation covers by X-band SAR images," IEEE Trans. Geosci. Remote Sensing, vol. 27, no. 6, pp. 709-718, Nov. 1989.

[8] Y. Inoue et al., "Season-long daily measurements of multifrequency (Ka, $\mathrm{Ku}, \mathrm{X}, \mathrm{C}$, and $\mathrm{L}$ ) and full-polarization backscatter signatures over paddy rice field and their relationship with biological variables," Remote Sensing of Environment, vol. 81, pp. 194-204, 2002.

[9] J. M. Lopez-Sanchez, S. R. Cloude, and J. D. Ballester-Berman, "Rice phenology monitoring by means of SAR polarimetry at X-band," IEEE Trans. Geosci. Remote Sensing, vol. 50, no. 7, pp. 2695-2709, July 2012.

[10] S. Yang, X. Zhao, B. Li, and G. Hua, "Interpreting RADARSAT-2 quadpolarization SAR signatures from rice paddy based on experiments," IEEE Geosci. Remote Sensing Letters, vol. 9, no. 1, pp. 65-69, Jan. 2012.

[11] K. Li, B. Brisco, Y. Shao, and R. Touzi, "Polarimetric decomposition with RADARSAT-2 for rice mapping and monitoring," Can. J. Remote Sensing, vol. 38, no. 2, pp. 169-179, 2012

[12] U. Meier, Ed., Growth Stages of Mono- and Dicotyledonous Plants. BBCH Monograph, 2nd edition, 2001, http://www.jki.bund.de/fileadmin/dam_uploads/_veroeff/bbch/BBCHSkala_englisch.pdf.

[13] J. C. Zadoks, T. T. Chang, and C. F. Konzak, "A decimal code for the growth stages of cereals," Weed Research, vol. 14, no. 6, pp. 415-421, Dec. 1974.

[14] I. Hajnsek, K. P. Papathanassiou, and S. R. Cloude, "Removal of additive noise in polarimetric eigenvalue processing," in Proceedings of IEEE IGARSS, Sydney, Australia, July 2001, vol. 6, pp. 2778-2780.

[15] C. Lopez-Martinez, E. Pottier, and S. R. Cloude, "Statistical assessment of eigenvector-based target decomposition theorems in radar polarimetry," IEEE Trans. Geosci. Remote Sensing, vol. 43, no. 9, pp. 2058-2074, Sept. 2005.

[16] S. R. Cloude and E. Pottier, "A review of target decomposition theorems in radar polarimetry," IEEE Trans. Geosci. Remote Sensing, vol. 34, no. 2, pp. 498-518, Mar. 1996.

[17] S. R. Cloude and E. Pottier, "An entropy based classification scheme for land applications of polarimetric SAR," IEEE Trans. Geosci. Remote Sensing, vol. 35, no. 1, pp. 68-78, Jan. 1997.

[18] A. Freeman and S. L. Durden, "A three-component scattering model for polarimetric SAR data," IEEE Trans. Geosci. Remote Sensing, vol. 36, no. 3, pp. 963-973, May 1998.

[19] J. J. van Zyl, Y. Kim, and M. Arii, "Requirements for model-based polarimetric decompositions," in Proc. IEEE Intl. Geosci. Remote Sensing Symp. (IGARSS), Boston, MA, U.S.A., July 2008, vol. 5, pp. 417-420.

[20] S. R. Cloude, Polarisation. Applications in remote sensing, Oxford University Press, 2009.

[21] R. K. Raney, "Hybrid-polarity SAR architecture," IEEE Trans. Geosci. Remote Sensing, vol. 45, no. 11, pp. 3397-3404, 2007.

[22] F. J. Charbonneau et al., "Compact polarimetry overview and applications assessment," Can. J. Remote Sensing, vol. 36, no. 2, 2010.

[23] J. D. Ballester-Berman and J. M. Lopez-Sanchez, "Time series of hybridpolarity parameters over agricultural crops," IEEE Geosci. Remote Sensing Letters, vol. 9, no. 1, pp. 139-143, Jan. 2012.

[24] S. R. Cloude, D. G. Goodenough, and H. Chen, "Compact decomposition theory," IEEE Geosci. Remote Sensing Letters, vol. 9, no. 1, pp. 28-32, Jan. 2012. 
TABLE VI

STATISTICS OF THE RETRIEVAL RESULTS FOR THE COMPACT-POL CASE.

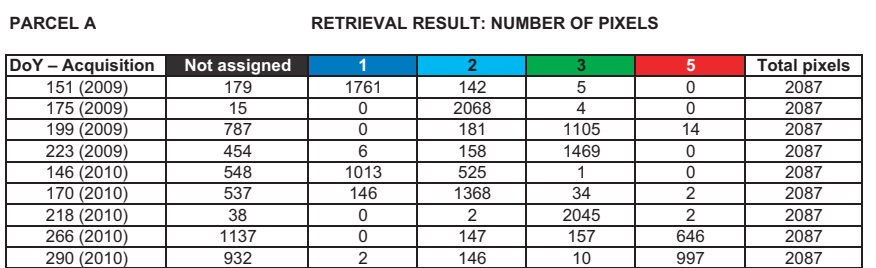

PARCEL B



PARCEL C

RETRIEVAL RESULT: NUMBER OF PIXELS



PARCEL D

RETRIEVAL RESULT: NUMBER OF PIXELS

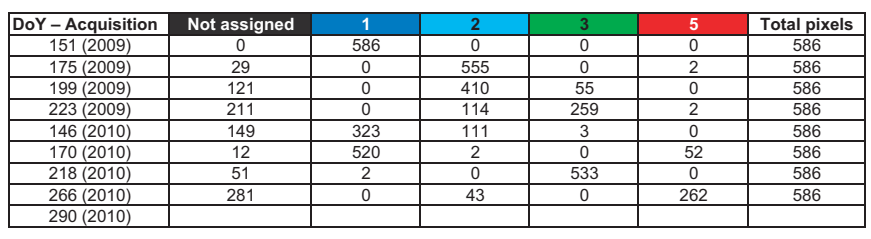

PARCEL E

RETRIEVAL RESULT: NUMBER OF PIXELS

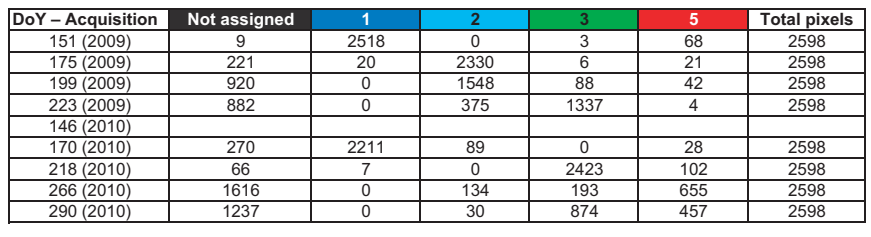

PARCEL F

RETRIEVAL RESULT: NUMBER OF PIXELS

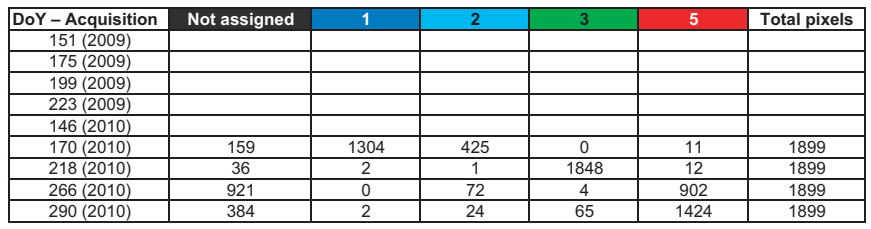

PERCENTAGE OVER ASSIGNED PIXELS

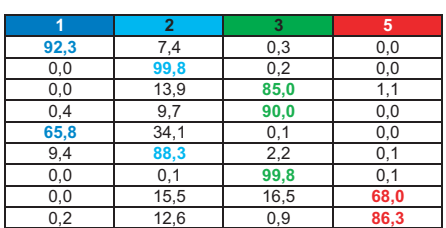

PERCENTAGE OVER ASSIGNED PIXELS

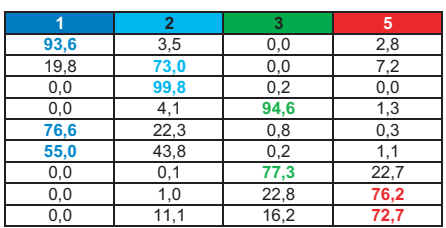

PERCENTAGE OVER ASSIGNED PIXELS

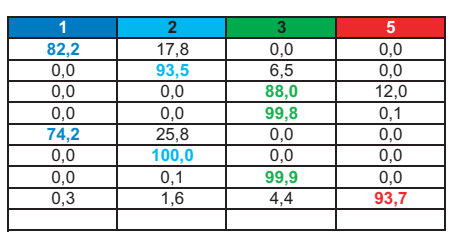

PERCENTAGE OVER ASSIGNED PIXELS

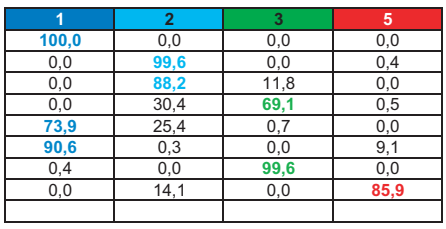

PERCENTAGE OVER ASSIGNED PIXELS

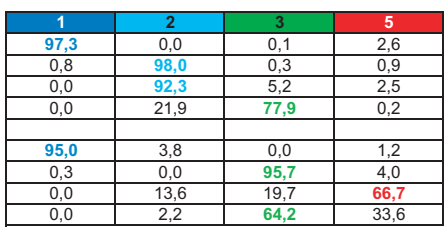

PERCENTAGE OVER ASSIGNED PIXELS



GROUND DATA

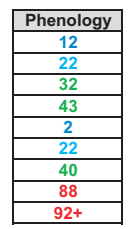

GROUND DATA

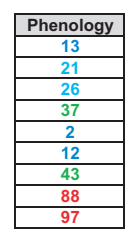

GROUND DATA

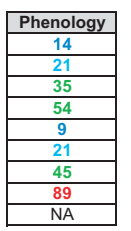

GROUND DATA

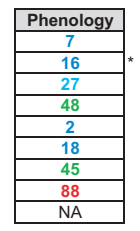

GROUND DATA



GROUND DATA

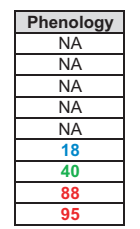

